\title{
Total Synthesis of Ingenol
}

Andrew Nickel, Toru Maruyama, Haifeng Tang, Prescott D. Murphy, ${ }^{\ddagger}$ Blake Greene, ${ }^{\ddagger}$

Naeem Yusuff, and John L. Wood*

Sterling Chemistry Laboratory, Department of Chemistry, Yale University, New Haven,

Connecticut 06520-8107

\section{Supplementary Information 1: Experimental Procedures and Tabular Characterization Data}

\section{Materials and Methods.}

Unless stated otherwise, reactions were performed in flame-dried glassware under a nitrogen atmosphere using freshly distilled solvents. Diethyl ether $\left(\mathrm{Et}_{2} \mathrm{O}\right)$ and tetrahydrofuran (THF) were distilled from sodium/benzophenone ketyl. Methylene chloride $\left(\mathrm{CH}_{2} \mathrm{Cl}_{2}\right)$, benzene $(\mathrm{PhH})$, toluene $(\mathrm{PhMe})$, triethylamine $\left(\mathrm{Et}_{3} \mathrm{~N}\right)$, pyridine, diisopropylamine, and piperidine were distilled from calcium hydride. Methyl sulfoxide (DMSO) and N,N-dimethylformamide (DMF) were either purchased from the Aldrich Chemical Company in Sure/Seal ${ }^{\mathrm{TM}}$ containers and used as received or stored over molecular sieves. All other commercially obtained reagents were used as received.

All reactions were magnetically stirred and monitored by thin-layer chromatography (TLC) using E. Merck silica gel $60 \mathrm{~F}_{254}$ precoated plates $(0.25 \mathrm{~mm})$. Column or flash chromatography was performed with the indicated solvents using silica gel (230-400 mesh) purchased from Silicycle. Concentration in vacuo refers to the removal of solvent with a Buchi R-3000 rotary evaporator at normal aspirator pressure followed by further evacuation with a two stage mechanical pump. 
All melting points were obtained on a Barnstead/Electrothermal 9100 melting point apparatus and are uncorrected. Infrared spectra were recorded on a Midac M1200 FTIR. ${ }^{1} \mathrm{H}$ and ${ }^{13} \mathrm{C}$ NMR spectra were recorded at room temperature unless otherwise noted on a Bruker AM-500, Bruker Avance DPX-500, or Bruker Avance DPX-400 spectrometer. Chemical shifts are reported relative to internal chloroform $\left({ }^{1} \mathrm{H}, \delta 7.26\right.$ ppm; $\left.{ }^{13} \mathrm{C}, \delta 77.23 \mathrm{ppm}\right)$ or pyridine $\left({ }^{1} \mathrm{H}, \delta 8.74,7.59\right.$, and $7.22 \mathrm{ppm} ;{ }^{13} \mathrm{C}, \delta 150.28$, 135.91, $123.90 \mathrm{ppm})$. Low resolution mass spectra were obtained on a Waters Micromass ${ }^{\circledR} \mathrm{ZQ}^{\mathrm{TM}}$ mass spectrometer using electrospray ionization (EI).

\section{Preparative Procedures.}

\section{Preparation of diene 5.}

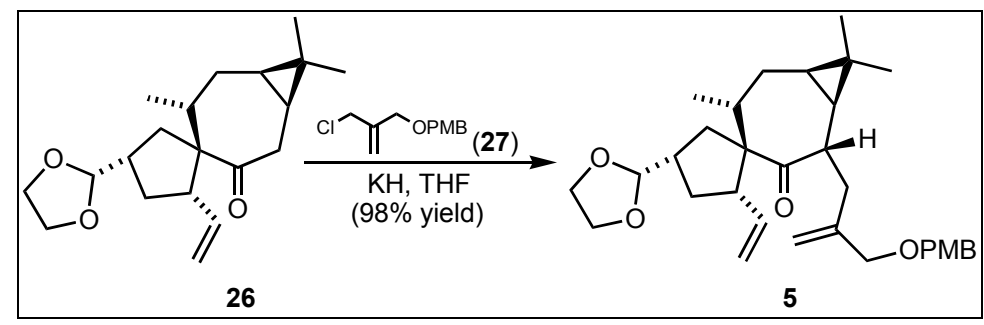

Diene 5. Ketone 26 was prepared as described in ref. 6e. Allyl chloride 27 was prepared following the procedure of Boulet, S. L.; Paquette, L. A. Synthesis 2002, 895900.

To a suspension of $\mathrm{KH}$ powder $(624 \mathrm{mg}, 15.6 \mathrm{mmol})$ in anhydrous THF $(40 \mathrm{ml})$ was added slowly a solution of ketone $26(2.5 \mathrm{~g}, 7.8 \mathrm{mmol})$ and allyl chloride $27(3.0 \mathrm{~g}$, $13 \mathrm{mmol})$ in THF (40 ml) at reflux under $\mathrm{N}_{2}$. After refluxing overnight, the reaction mixture was cooled to room temperature and treated with $\mathrm{EtOH}(2 \mathrm{ml})$ to quench excess $\mathrm{KH}$. The solution was poured into dilute $\mathrm{HCl}$ and extracted with ether. The organic layer was washed with water, brine, and dried over $\mathrm{Na}_{2} \mathrm{SO}_{4}$. Concentration and purification by 
flash chromatography (hexane:EtOAc 6:1 $\rightarrow 4: 1$ eluent) gave diene 5 (3.9 g, 98\% yield) as a white solid.

Diene 5: m.p. $104^{\circ} \mathrm{C}$; FTIR (thin film/NaCl) 2959 (m), 2867 (m), 1683 (s), 1514 (m), 1245 (s), $1088(\mathrm{~m}) \mathrm{cm}^{-1} ;{ }^{1} \mathrm{H}$ NMR (500 MHz, $\left.\mathrm{CDCl}_{3}\right) \delta 7.22(\mathrm{~d}, J=8 \mathrm{~Hz}, 2 \mathrm{H}), 6.84$ (d, $J=8 \mathrm{~Hz}, 2 \mathrm{H}), 5.59$ (dt, $J=10,18,18 \mathrm{~Hz}, 1 \mathrm{H}), 5.10(\mathrm{br} \mathrm{s}, 1 \mathrm{H}), 4.96(\mathrm{~d}, J=17 \mathrm{~Hz}$, 1H), $4.90(\mathrm{~d}, J=10 \mathrm{~Hz}, 1 \mathrm{H}), 4.89$ (s, 1H), 4.75 (d, $J=1 \mathrm{~Hz}, 1 \mathrm{H}), 4.39$ (s, 2H), 3.94-3.89 (m, 2H), 3.84-3.81 (m, 2H), $3.78(\mathrm{~s}, 2 \mathrm{H}), 3.76(\mathrm{~s}, 3 \mathrm{H}), 2.70(\mathrm{~m}, 1 \mathrm{H}), 2.35-2.25(\mathrm{~m}, 2 \mathrm{H})$, 2.16-2.13 (m, 2H), 1.96 (dd, $J=6,14 \mathrm{~Hz}, 1 \mathrm{H}), 1.89-1.75(\mathrm{~m}, 3 \mathrm{H}), 1.58(\mathrm{dd}, J=10,14$ Hz, 1H), 1.45 (m, 1H), 0.92 (s, 3H), 0.89 (d, $J=7 \mathrm{~Hz}, 3 \mathrm{H}), 0.86$ (s, 3H), 0.62 (dd, $J=9$, $15 \mathrm{~Hz}, 1 \mathrm{H}), 0.07(\mathrm{t}, J=9 \mathrm{~Hz}, 1 \mathrm{H}) ;{ }^{13} \mathrm{C} \mathrm{NMR}\left(100 \mathrm{MHz}, \mathrm{CDCl}_{3}\right) \delta 211.9,159.1,143.7$, $140.4,130.5,129.3,115.4,113.8,113.4,107.2,72.5,71.8,68.4,65.2,65.1,55.3,49.3$, $46.8,39.2,34.5,34.5,32.4,30.8,28.7,27.6,26.8,22.6,21.0,15.9,14.8$; LRMS (EI) $\mathrm{m} / \mathrm{z}$ $531.4\left(\mathrm{~m}+\mathrm{Na}^{+}\right)$.

\section{Preparation of tetracycle 6.}

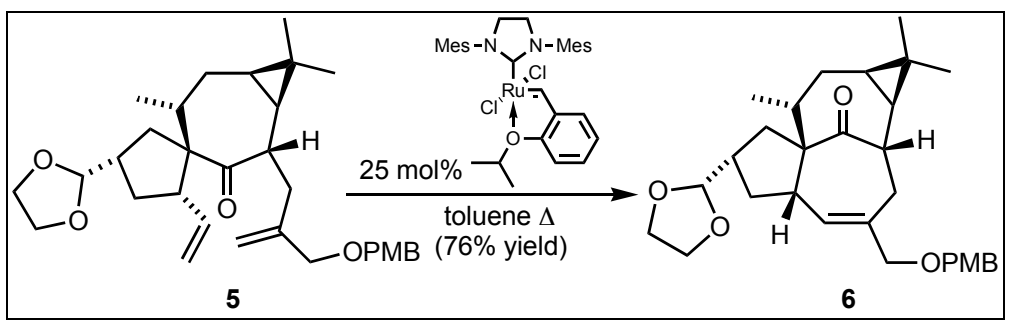

Tetracycle 6. To a solution of diene 5 (400 $\mathrm{mg}, 787 \mu \mathrm{mol})$ in toluene (40 ml) was added the Hoveyda-Grubbs $2^{\text {nd }}$ generation catalyst $(123 \mathrm{mg}, 200 \mu \mathrm{mol})$ at room temperature under a positive pressure of $\mathrm{N}_{2}$. The reaction mixture was stirred under reflux for $30 \mathrm{hrs}$ with bubbling of $\mathrm{N}_{2}$ through a needle. The reaction mixture was cooled 
to room temperature and directly subjected to column chromatography on silica gel $(0: 1 \rightarrow 4: 1$ hexnae:EtOAc) to give tetracycle 6 ( $290 \mathrm{mg}, 76 \%$ yield) as a green oil.

Tetracycle 6: FTIR (thin film/NaCl) 2951 (s), 1720 (s), 1612 (m), 1585 (w), 1513 (s), 1248 (s), 733 (s) $\mathrm{cm}^{-1} ;{ }^{1} \mathrm{H}$ NMR (500 MHz, $\left.\mathrm{CDCl}_{3}\right) \delta 7.24$ (d, $\left.J=8 \mathrm{~Hz}, 2 \mathrm{H}\right)$, $6.86(\mathrm{~d}, J=8 \mathrm{~Hz}, 2 \mathrm{H}), 5.38(\mathrm{br} \mathrm{s}, 1 \mathrm{H}), 4.68(\mathrm{~d}, J=5 \mathrm{~Hz}, 1 \mathrm{H}), 4.32(\mathrm{~d}, J=11.5 \mathrm{~Hz}, 1 \mathrm{H})$, $4.28(\mathrm{~d}, J=11.5 \mathrm{~Hz}, 1 \mathrm{H}), 3.96-3.91(\mathrm{~m}, 2 \mathrm{H}), 3.85-3.8(\mathrm{~m}, 2 \mathrm{H}), 3.79(\mathrm{~s}, 3 \mathrm{H}), 3.78(\mathrm{~s}, 2 \mathrm{H})$, 3.25-3.20 (m, 2H), 2.40 (br t, $J=14 \mathrm{~Hz}, 1 \mathrm{H}), 2.28-2.17(\mathrm{~m}, 3 \mathrm{H}), 2.02(\mathrm{~m}, 1 \mathrm{H}), 1.91-1.78$ (m, 3H), $1.72($ br t, $J=11.5 \mathrm{~Hz}, 1 \mathrm{H}), 1.46(\mathrm{~m}, 1 \mathrm{H}), 1.11(\mathrm{~s}, 3 \mathrm{H}), 1.03(\mathrm{~s}, 3 \mathrm{H}), 0.98(\mathrm{~d}, J=$ $7 \mathrm{~Hz}, 3 \mathrm{H}), 0.71-0.64(\mathrm{~m}, 2 \mathrm{H}) ;{ }^{13} \mathrm{C} \mathrm{NMR}\left(125 \mathrm{MHz}, \mathrm{CDCl}_{3}\right) \delta$ 212.4, 159.3, 138.9, 135.8, 130.7, 129.7, 114.0, 107.3, 76.2, 71.9, 65.3, 65.2, 55.4, 45.2, 44.7, 42.0, 36.3, 34.9, 32.3, 29.5, 28.8, 23.8, 23.7, 23.3, 15.9, 15.4; LRMS (EI) $m / z 503.4\left(\mathrm{~m}+\mathrm{Na}^{+}\right)$.

\section{Preparation of diene 7.}

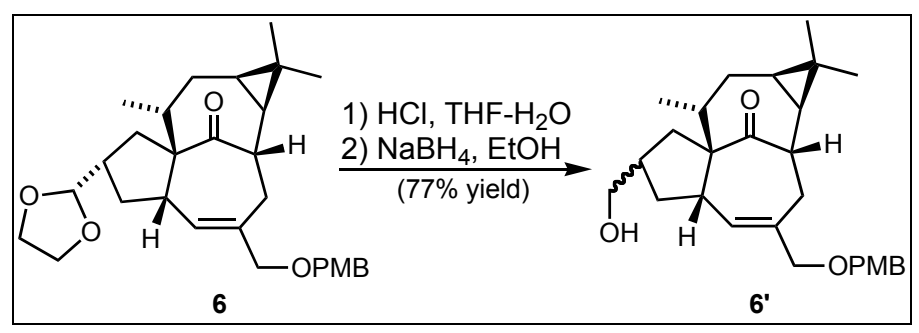

Diene 7. A mixture of dioxolane $6(2.7 \mathrm{~g}, 5.6 \mathrm{mmol})$ and $1 \mathrm{~N} \mathrm{HCl}(28 \mathrm{~mL}, 28$ mmol) in THF ( $84 \mathrm{~mL})$ was stirred at reflux for $3 \mathrm{hrs}$. The reaction mixture was cooled, poured into water, and extracted with EtOAc and hexane. The organic layer was washed with water and brine, and dried over $\mathrm{Na}_{2} \mathrm{SO}_{4}$. The solvent was removed in vacuo and the residual oil was dissolved in ethanol $(30 \mathrm{~mL})$ and THF $(20 \mathrm{~mL})$. The solution was cooled to $0^{\circ} \mathrm{C}$ before addition of $\mathrm{NaBH}_{4}(318 \mathrm{mg}, 8.4 \mathrm{mmol})$. After stirring for 15 minutes, the reaction was quenched with $\mathrm{H}_{2} \mathrm{O}$. The mixture was extracted with EtOAc, washed with 
brine, and dried over $\mathrm{Na}_{2} \mathrm{SO}_{4}$. The solvent was removed in vacuo and the residue was purified by flash chromatography $(3: 1 \rightarrow 1: 1$ hexane:EtOAc eluent) to give a mixture of diastereomeric alcohols 6' (1.9 g, 77\% over two steps) as a colorless oil.

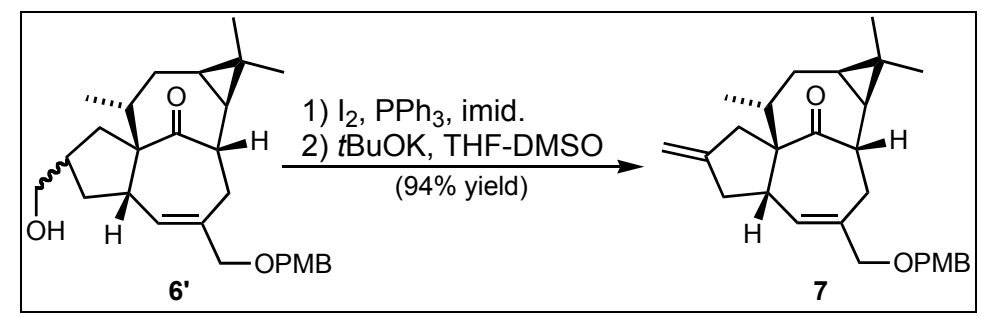

To a solution of alcohol 6' (582 mg, $1.33 \mathrm{mmol})$, triphenylphosphine (453 mg, $1.73 \mathrm{mmol})$ and imidazole $(181 \mathrm{mg}, 2.66 \mathrm{mmol})$ in anhydrous THF was added iodine (404 mg, $1.59 \mathrm{mmol}$ ) at $0^{\circ} \mathrm{C}$ under an atmosphere of $\mathrm{N}_{2}$. After stirring for $3 \mathrm{hrs}$, the black reaction mixture was diluted with ether and shaken with aqueous $\mathrm{Na}_{2} \mathrm{~S}_{2} \mathrm{O}_{3}$. The organic layer was washed with water and brine, and dried over $\mathrm{Na}_{2} \mathrm{SO}_{4}$. The solvent was removed in vacuo and the residual suspension was dissolved in anhydrous THF $(10 \mathrm{~mL})$. The solution was tranferred via cannula to a solution of $t$ - BuOK (596 mg, $5.32 \mathrm{mmol})$ in anhydrous DMSO $(10 \mathrm{~mL})$ at room temperature under $\mathrm{N}_{2}$. The reaction was stirred overnight and then diluted with ether. The mixture was washed with water and brine, and dried over $\mathrm{Na}_{2} \mathrm{SO}_{4}$. The solvent was removed in vacuo and the residue was purified by flash chromatography (1:0 $\rightarrow 3: 1$ hexane:EtOAC eluent) to afford diene 7 (527 mg, 94\% in two steps as a colorless oil.

Diene 7: FTIR (thin film/NaCl) 2916 (m), 1722 (s), 1612 (m), 1514 (s), 1248 (s), $1072(\mathrm{~m}), 1036(\mathrm{~m}) \mathrm{cm}^{-1} ;{ }^{1} \mathrm{H}$ NMR $\left(400 \mathrm{MHz}, \mathrm{CDCl}_{3}\right) \delta 7.23$ (d, $\left.J=9,2 \mathrm{H}\right), 6.86(\mathrm{~d}, J=$ $9 \mathrm{~Hz}, 2 \mathrm{H}), 5.25$ (br s, 1H), $4.81(\mathrm{~s}, 1 \mathrm{H}), 4.80(\mathrm{~s}, 1 \mathrm{H}), 4.30$ (d, $J=11.5 \mathrm{~Hz}, 1 \mathrm{H}), 4.24$ (d, $J$ $=11.5 \mathrm{~Hz}, 1 \mathrm{H}), 3.79(\mathrm{~s}, 3 \mathrm{H}), 3.79(\mathrm{~d}, J=11.5 \mathrm{~Hz}, 1 \mathrm{H}), 3.75(\mathrm{~d}, J=11.5 \mathrm{~Hz}, 1 \mathrm{H}), 3.30-$ $3.23(\mathrm{~m}, 2 \mathrm{H}), 2.74(\mathrm{dd}, J=2,17 \mathrm{~Hz}, 1 \mathrm{H}), 2.65(\mathrm{dd}, J=8,15 \mathrm{~Hz}, 1 \mathrm{H}), 2.44-2.14(\mathrm{~m}, 4 \mathrm{H})$, 
2.02-1.99 (m, 1H), $1.90(\mathrm{ddd}, J=5,5,13 \mathrm{~Hz}, 1 \mathrm{H}), 1.83-1.76(\mathrm{~m}, 1 \mathrm{H}), 1.12(\mathrm{~s}, 3 \mathrm{H}), 1.01$ (s, 3H), $0.96(\mathrm{~d}, J=7 \mathrm{~Hz}, 3 \mathrm{H}), 0.74-0.63(\mathrm{~m}, 2 \mathrm{H}) ;{ }^{13} \mathrm{C} \mathrm{NMR}\left(100 \mathrm{MHz}, \mathrm{CDCl}_{3}\right) \delta 211.3$, $159.5,150.4,140.7,134.7,130.7,129.7,114.1,106.1,76.0,72.0,71.4,55.5,44.8,44.5$, $40.7,37.2,34.2,32.6,29.3,28.8,23.9,23.7,23.3,15.6,15.4$; LRMS (EI) $m / z 443.3(\mathrm{~m}+$ $\mathrm{Na}^{+}$.

\section{Preparation of allylic alcohol 8.}

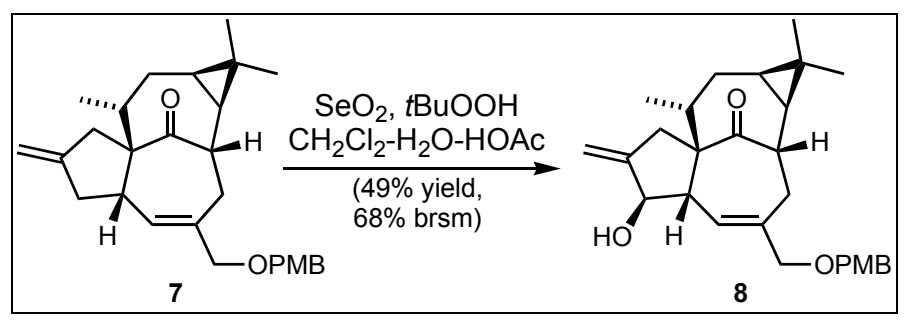

Allylic alcohol 8. To a vigorously stirred solution of diene 7 (527 mg, 1.25 mmol) in $\mathrm{CH}_{2} \mathrm{Cl}_{2}(28 \mathrm{ml})$ was added water $(280 \mu \mathrm{L})$, acetic acid $(45 \mu \mathrm{L})$, and selenium dioxide ( $25 \mathrm{mg}, 0.23 \mathrm{mmol}, 18 \mathrm{~mol} \%$ ). The suspension was stirred at room temperature for $10 \mathrm{~min}$ before being cooled to $0^{\circ} \mathrm{C}$, when $t$ - $\mathrm{BuOOH}(5-6 \mathrm{M}$ in decane, $640 \mu \mathrm{L}, 3.5$ mmol, 2.5 equiv) was added dropwise. The cold bath was removed, and the reaction allowed to proceed at room temperature for $2_{-} \mathrm{hrs}$, at which time the reaction was quenched with dilute aqueous $\mathrm{KOH}$ and extracted with ether. The organic phase was washed with water, brine, and dried over $\mathrm{MgSO}_{4}$. The organic phase was concentrated in vacuo and chromatographed on silica gel (hexane:EtOAc 9:1 $\rightarrow 6: 1$ eluent) to afford starting diene 7 (155 mg, 30\%) and allylic alcohol 8 (265 mg, 49\%) as a colorless oil.

Allylic alcohol 8: FTIR (thin film/NaCl) 2934 (m), 1721 (s), 1612 (w), 1513 (s), 1248, (s), 1036 (s) $\mathrm{cm}^{-1} ;{ }^{1} \mathrm{H}$ NMR (500 MHz, $\left.\mathrm{CDCl}_{3}\right) \delta 7.22$ (br d, $\left.J=8.5 \mathrm{~Hz}, 2 \mathrm{H}\right), 6.86$ (br d, $J=8.5 \mathrm{~Hz}, 2 \mathrm{H}), 5.19$ (br s, 1H), 5.09 (s, 1H), 4.99 (s, 1H), 4.30 (d, $J=11.5 \mathrm{~Hz}$, 
1H) $4.25(\mathrm{~d}, J=11.5 \mathrm{~Hz}, 1 \mathrm{H}), 4.20(\mathrm{~s}, 1 \mathrm{H}), 3.80(\mathrm{~s}, 3 \mathrm{H}), 3.77(\mathrm{~s}, 2 \mathrm{H}), 3.23-3.15(\mathrm{~m}, 2 \mathrm{H})$, 2.86 (d, $J=17.5 \mathrm{~Hz}, 1 \mathrm{H}), 2.56-2.52$ (m, 1h), 2.49 (d, $J=17.5 \mathrm{~Hz}, 1 \mathrm{H}), 2.39$ (t, $J=16 \mathrm{~Hz}$, 1H), $2.21(\mathrm{~d}, J=17 \mathrm{~Hz}, 1 \mathrm{H}), 1.93-1.80(\mathrm{~m}, 2 \mathrm{H}), 1.8-1.6(\mathrm{br} \mathrm{s}, 1 \mathrm{H}), 1.12(\mathrm{~s}, 3 \mathrm{H}), 1.04(\mathrm{~s}$, $3 \mathrm{H}), 0.96(\mathrm{~d}, J=7 \mathrm{~Hz}, 3 \mathrm{H}), 0.73-0.68(\mathrm{~m}, 1 \mathrm{H}), 0.64(\mathrm{dd}, J=8,12 \mathrm{~Hz}, 1 \mathrm{H}) ;{ }^{13} \mathrm{C} \mathrm{NMR}$ $\left(125 \mathrm{MHz}, \mathrm{CDCl}_{3}\right) \delta$ 2 210.9, 159.4, 153.0, 141.2, 130.4, 129.8, 129.7, 114.0, 109.8, 83.5, 75.7, 71.3, 55.5, 52.3, 44.9, 38.0, 32.7, 32.5, 28.8, 23.7, 23.2, 23.1, 15.7, 15.4; LRMS (EI) $m / z 459.3\left(\mathrm{~m}+\mathrm{Na}^{+}\right)$.

\section{Preparation of Exo-enone 8'.}

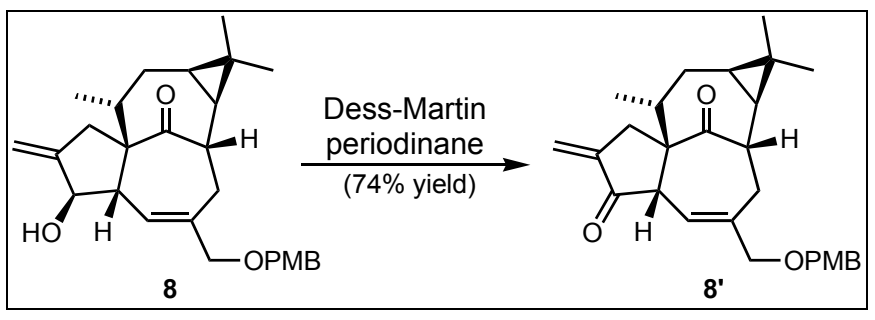

Exo-enone 8'. A stirred solution of allylic alcohol 8 (174 mg, $400 \mu \mathrm{mol})$ in $\mathrm{CH}_{2} \mathrm{Cl}_{2}(5 \mathrm{~mL})$ in an open flask was cooled to $-40^{\circ} \mathrm{C}$ in an $\mathrm{CH}_{3} \mathrm{CN} / \mathrm{CO}_{2}$ bath. DessMartin periodinane ( $215 \mathrm{mg}, 510 \mu \mathrm{mol}, 1.25$ equiv) was then added. The reaction was allowed to warm to $10^{\circ} \mathrm{C}$ over $90 \mathrm{~min}$, at which time the reaction was quenched with $\mathrm{Na}_{2} \mathrm{~S}_{2} \mathrm{O}_{3}(10 \%$ aqueous solution, $10 \mathrm{~mL})$. The mixture was stirred vigorously until the two layers were clear. The crude product was extracted with $\mathrm{CH}_{2} \mathrm{Cl}_{2}$, washed with brine, dried over $\mathrm{MgSO}_{4}$, and filtered. Concentration followed by flash chromatography (12:1 hexanes:EtOAc eluent) afforded exo-enone 8' (128 mg, 74\% yield) as a colorless oil.

Exo-enone 8': FTIR (thin film/ $\mathrm{NaCl}$ ) 2960 (m), 1725 (s), 1612 (m), 1514 (s), $1250(\mathrm{~s}) \mathrm{cm}^{-1} ;{ }^{1} \mathrm{H} \mathrm{NMR}\left(500 \mathrm{MHz}, \mathrm{CDCl}_{3}, 50{ }^{\circ} \mathrm{C}\right) \delta 7.21(\mathrm{~d}, J=8.5 \mathrm{~Hz}, 2 \mathrm{H}), 6.86(\mathrm{~d}, J=$ $8.5 \mathrm{~Hz}, 2 \mathrm{H}), 6.03(\mathrm{br} \mathrm{s}, 1 \mathrm{H}), 5.41(\mathrm{~s}, 1 \mathrm{H}), 5.36(\mathrm{~s}, 1 \mathrm{H}), 4.33(\mathrm{~d}, J=12 \mathrm{~Hz}, 1 \mathrm{H}), 4.29$ (d, $J$ 
$=12 \mathrm{~Hz}), 3.82(\mathrm{~s}, 2 \mathrm{H}), 3.80(\mathrm{~s}, 3 \mathrm{H}), 3.44(\mathrm{~s}, 1 \mathrm{H}), 3.13(\mathrm{dt}, J=3.0,12,12 \mathrm{~Hz}, 1 \mathrm{H}), 2.94$ (br d, $J=18 \mathrm{~Hz}, 1 \mathrm{H}), 2.85$ (br s, $J=18 \mathrm{~Hz}, 1 \mathrm{H}), 2.47$ ( br t, $J=14 \mathrm{~Hz}, 1 \mathrm{H}), 2.26$ (br d, $J$ $=16 \mathrm{~Hz}, 1 \mathrm{H}), 2.00(\mathrm{~m}, 1 \mathrm{H}), 1.93(\mathrm{dt}, J=5,5,14 \mathrm{~Hz}, 1 \mathrm{H}), 1.80(\mathrm{~m}, 1 \mathrm{H}), 1.14(\mathrm{~s}, 3 \mathrm{H})$, $1.07(\mathrm{~s}, 3 \mathrm{H}), 1.02(\mathrm{~d}, J=15 \mathrm{~Hz}, 3 \mathrm{H}), 0.74(\mathrm{~m}, 2 \mathrm{H}) ;{ }^{13} \mathrm{C}$ NMR $\left(125 \mathrm{MHz}, \mathrm{CDCl}_{3}, 50{ }^{\circ} \mathrm{C}\right)$ $\delta$ $210.5,205.3,159.7,144.6,143.9,130.6,129.7,124.0,118.9,114.3,75.8,71.9,65.4$, 55.9, 55.5, 45.4, 37.1, 32.4, 31.5, 29.1, 28.9, 24.2, 23.8, 23.6, 15.8, 15.3; LRMS (EI) $\mathrm{m} / \mathrm{z}$ $457.3\left(\mathrm{~m}+\mathrm{Na}^{+}\right)$.

\section{Preparation of Cyclopentenone 9.}

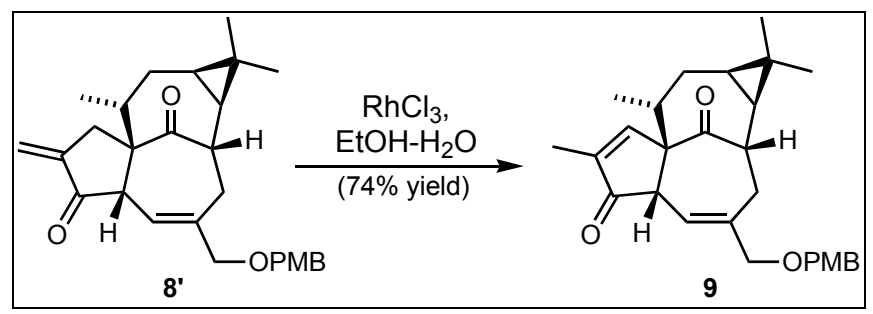

Cyclopentenone 9. To a stirred solution of exo-enone 8' $(125 \mathrm{mg}, 285 \mu \mathrm{mol})$ in 95\% ethanol ( $15 \mathrm{~mL}$ ) was added $\mathrm{RhCl}_{3} \mathrm{xH}_{2} \mathrm{O}$ (38\% rhodium, $\left.23 \mathrm{mg}, 85 \mu \mathrm{mol}, 30 \mathrm{~mol} \%\right)$. The reaction mixture was heated in a sealed tube to $115^{\circ} \mathrm{C}$ for $35 \mathrm{~min}$. After the solution was cooled to rt, it was filtered through a celite pad. Concentration in vacuo followed by flash chromatography (11:1 hexanes:EtOAc eluent) afforded cyclopentenone 9 (93 mg, $74 \%$ yield) as a colorless oil.

Cyclopentenone 9: FTIR (thin film/NaCl) 2199 (w), 1710 (s), 1612 (w), 1513 (m), $1248(\mathrm{~m}) ;{ }^{1} \mathrm{H}$ NMR (500 MHz, $\left.\mathrm{CDCl}_{3}\right) \delta 7.59$ (br s, $\left.1 \mathrm{H}\right), 7.20$ (br dt, $\left.J=8 \mathrm{~Hz}, 2 \mathrm{H}\right)$, $6.86(\mathrm{ddd}, J=2,3,9 \mathrm{~Hz}, 2 \mathrm{H}), 5.46(\mathrm{br} \mathrm{s}, 1 \mathrm{H}), 4.28(\mathrm{~s}, 2 \mathrm{H}), 3.81(\mathrm{~s}, 2 \mathrm{H}), 3.79(\mathrm{~s}, 3 \mathrm{H})$, 3.26 (br s, 1H), 3.09-3.02 (m, 1H), 2.39 (br t, $J=15 \mathrm{~Hz}, 1 \mathrm{H}), 2.24$ (br d, $J=17 \mathrm{~Hz}, 1 \mathrm{H}$ ), 1.98-1.85 (m, 3H), 1.79 (d, $J=1 \mathrm{~Hz}, 3 \mathrm{H}), 1.13$ (s, 3H), 1.05 (s, 3H), 1.04 (d, $J=6 \mathrm{~Hz}$, 
3H), 0.80-0.76 (m, 2H); ${ }^{13} \mathrm{C}$ NMR (125 MHz, $\left.\mathrm{CDCl}_{3}\right) \delta$ 209.2, 207.2, 159.4, 158.1, $142.1,140.8,130.3,129.7,125.7,114.0,76.0,71.6,70.3,55.5,54.1,45.3,42.7,31.4$, 31.0, 28.9, 23.8, 23.6, 23.2, 17.0, 15.3, 10.8; LRMS (EI) $m / z 457.3\left(\mathrm{~m}+\mathrm{Na}^{+}\right)$.

\section{Preparation of alcohol 10.}

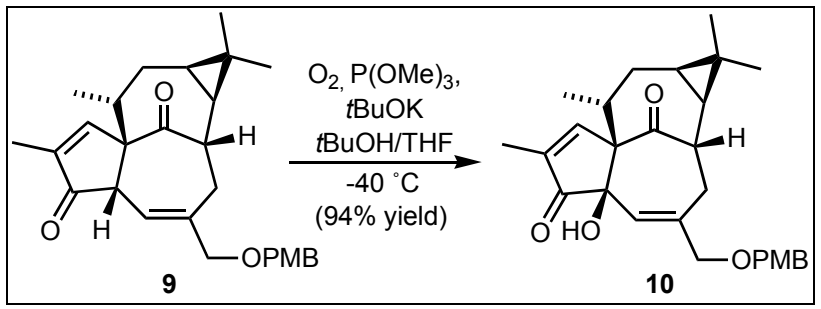

Alcohol 10. To a stirred solution of cyclopentenone $9(78 \mathrm{mg}, 180 \mu \mathrm{mol})$ in $\mathrm{THF} / t$-BuOH (4:1, $10 \mathrm{~mL}$ total) was added $\mathrm{P}(\mathrm{OMe})_{3}(40 \mu \mathrm{L}, 340 \mu \mathrm{mol}, 1.9$ equiv) via syringe. The solution was cooled to $-40^{\circ} \mathrm{C}$ with a $\mathrm{CH}_{3} \mathrm{CN} / \mathrm{CO}_{2}$ bath. After bubbling $\mathrm{O}_{2}$ through the solution for 5 minutes, solid $t$-BuOK ( $82 \mathrm{mg}, 730 \mu \mathrm{mol}, 4$ equiv) was added at once, at which time the solution immediately changed from clear to bright yellow. The reaction mixture was allowed to stir at $-40^{\circ} \mathrm{C}$ with bubbling $\mathrm{O}_{2}$ for $45 \mathrm{~min}$. The reaction was then quenched with $700 \mu \mathrm{L} 1 \mathrm{~N} \mathrm{HCl}$, allowed to warm to room temperature, extracted with $\mathrm{Et}_{2} \mathrm{O}$, washed with brine, and dried over $\mathrm{MgSO}_{4}$. Concentration in vacuo followed by flash chromatography $(15: 1 \rightarrow 10: 1$ hexanes:EtOAc eluent) afforded alcohol 10 (76 $\mathrm{mg}, 94 \%$ yield) as a white solid.

Alcohol 10: m.p. $113-114^{\circ} \mathrm{C}$; FTIR (thin film/NaCl) 3430 (br w), 2923 (m), 1720 (s), 1712 (s), $1513(\mathrm{~m}), 1249$ (m) $\mathrm{cm}^{-1} ;{ }^{1} \mathrm{H}$ NMR $\left(500 \mathrm{MHz}, \mathrm{CDCl}_{3}\right) \delta 7.66(\mathrm{~d}, J=1.5$ Hz, 1H), 7.19 (dt, $J=9 \mathrm{~Hz}, 2 \mathrm{H}), 6.86(\mathrm{dt}, J=9,2 \mathrm{H}), 5.44$ (br s, $1 \mathrm{H}), 4.31$ (d, $J=11.5$ Hz, 1H), 4.27 (d, $J=11.5 \mathrm{~Hz}, 1 \mathrm{H}), 3.80$ (s, 3H), 3.79 (s, 2H), 3.46 (ddd $J=3,10,14 \mathrm{~Hz}$, 1H), $3.13($ br s, 1H), $2.43(\mathrm{dd}, J=1.5,13 \mathrm{~Hz}, 1 \mathrm{H}), 2.40(\mathrm{dd}, J=1.5,13 \mathrm{~Hz}, 1 \mathrm{H}), 2.40$ 
(dd, $J=1.5,16 \mathrm{~Hz}, 1 \mathrm{H}), 2.29(\mathrm{dd}, J=1.5,16 \mathrm{~Hz}, 1 \mathrm{H}), 2.17-2.14(\mathrm{~m}, 1 \mathrm{H}), 1.84(\mathrm{~d}, J=1.5$ Hz, 3H), 1.83-1.78 (m, 1H), 1.15 (s, 3H), 1.07 (d, $J=7$ Hz, 3H), 1.06 (s, 3H), 0.75-0.72 (m, 2H); ${ }^{13} \mathrm{C}$ NMR $\left(125 \mathrm{MHz}, \mathrm{CDCl}_{3}\right) \delta$ 208.9, 208.3, 159.5, 158.6, 146.6, 137.9, 130.2, $129.7,128.2,114.0,79.3,75.3,72.4,71.9,55.5,43.9,39.8,32.4,30.8,28.8,24.3,24.2$, 23.1, 17.6, 15.4, 10.9; LRMS (EI) $m / z 473.3\left(\mathrm{~m}+\mathrm{Na}^{+}\right)$.

\section{Preparation of TMS ether 11.}

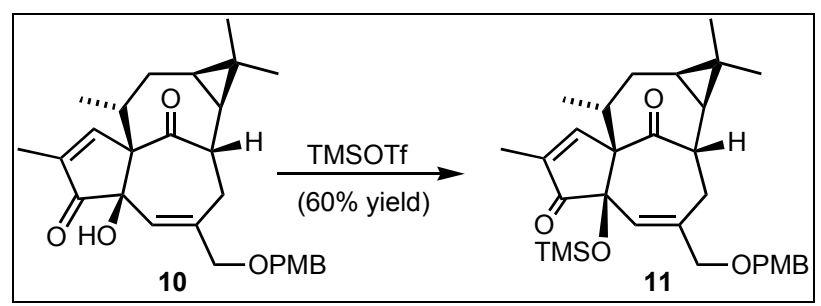

TMS ether 11. To a stirred solution of alcohol 10 (20 mg, $44 \mu \mathrm{mol})$ in $\mathrm{CH}_{2} \mathrm{Cl}_{2}$ (700 $\mu \mathrm{L})$ was added triethylamine $(500 \mu \mathrm{L}, 3.5 \mathrm{mmol}, 80$ equiv). The solution was cooled to $0^{\circ} \mathrm{C}$ in a ice bath and TMSOTf $(17 \mu \mathrm{L}, 88 \mu \mathrm{mol}, 2$ equiv) was added over 30 seconds via syringe. The reaction was stirred at $0^{\circ} \mathrm{C}$ for $15 \mathrm{~min}$, at which time the reaction was quenched with aqueous $\mathrm{NaHCO}_{3}$ and extracted with $\mathrm{CH}_{2} \mathrm{Cl}_{2}$. The organic phase was washed with brine, dried over $\mathrm{MgSO}_{4}$, and filtered. Following concentration in vacuo, flash chromatography on silica gel (15:1 hexane:EtOAc eluent) afforded TMS ether $11(14 \mathrm{mg}, 60 \%)$ as a colorless oil. 


\section{Reduction of $\alpha$-hydroxyketone 10 .}

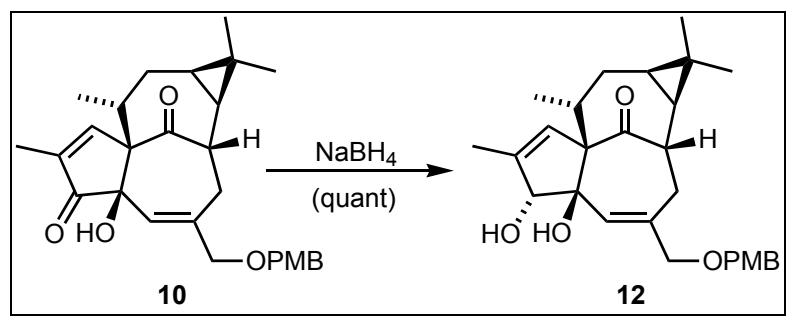

Reduction of $\boldsymbol{\alpha}$-hydroxyketone $\mathbf{1 0}$. To a stirred solution of enone $\mathbf{1 0}$ (5 mg, 11 $\mu \mathrm{mol})$ in $\mathrm{MeOH}(1 \mathrm{~mL})$ in an ice bath was added $\mathrm{NaBH}_{4}(1 \mathrm{mg}, 25 \mu$ mol, 2 equiv) at once. After $5 \mathrm{~min}$, aqueous $\mathrm{HCl}(1 \mathrm{~N}, 500 \mu \mathrm{L})$ was added to the reaction and stirred for an additional 5 min before extracting with EtOAc. The organic phase was washed with sat'd $\mathrm{NaHCO}_{3}$ and brine, and concentrated in vacuo. The resulting oil was azeotroped with $\mathrm{MeOH}(3 \times 10 \mathrm{~mL})$ to give diol 12 as a crude oil. Flash chromatography (3:1 hexanes:EtOAc eluent) afforded diol $\mathbf{1 2}(5 \mathrm{mg}, 99 \%)$ as a colorless oil. 
Diol 12: FTIR (thin film/NaCl) 3420 (br), 2921 (s), 1719 (s), 1612 (m), 1513 (s), 1249 (s), $1036(\mathrm{~m}) \mathrm{cm}^{-1} ;{ }^{1} \mathrm{H}$ NMR (400 MHz, $\left.\mathrm{CDCl}_{3}\right) \delta 7.23($ br d, $J=9 \mathrm{~Hz}, 2 \mathrm{H}), 6.86$ (br d, $J=9 \mathrm{~Hz}, 2 \mathrm{H}), 6.28(\mathrm{~s}, 1 \mathrm{H}), 5.81(\mathrm{t}, J=1.5 \mathrm{~Hz}, 1 \mathrm{H}), 4.78(\mathrm{~s}, 1 \mathrm{H}), 4.36(\mathrm{~d}, J=11.5$ Hz, 1H), 4.29 (d, J=11.5 Hz, 1H), $3.83(\mathrm{~s}, 2 \mathrm{H}), 3.80(\mathrm{~s}, 3 \mathrm{H}), 3.79-3.72(\mathrm{~m}, 1 \mathrm{H}), 2.45-$ $2.30(\mathrm{~m}, 5 \mathrm{H}), 1.80-1.72(\mathrm{~m}, 1 \mathrm{H}), 1.72(\mathrm{~s}, 3 \mathrm{H}), 1.11(\mathrm{~s}, 3 \mathrm{H}), 1.04(\mathrm{~s}, 3 \mathrm{H}), 0.94(\mathrm{~d}, J=7$ $\mathrm{Hz}, 3 \mathrm{H}), 0.73-0.66(\mathrm{~m}, 2 \mathrm{H}) ;{ }^{13} \mathrm{C}$ NMR $\left(100 \mathrm{MHz}, \mathrm{CDCl}_{3}\right) \delta 211.2,159.4,150.0,138.9$, $130.3,129.9,128.4,125.8,114.0,84.8,83.7,76.4,72.2,72.1,55.5,41.3,35.9,32.9,30.3$, 28.8, 24.2, 24.2, 23.2, 17.7, 15.6, 13.9; LRMS (EI) $m / z 475.3\left(\mathrm{~m}+\mathrm{Na}^{+}\right)$.

\section{Reduction of $\alpha$-silyloxyketone 11 .}

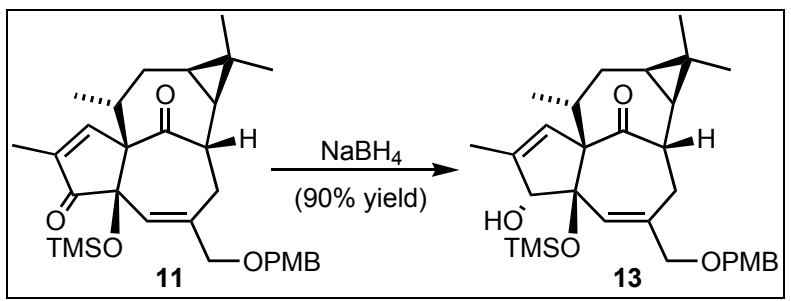


Reduction of $\boldsymbol{\alpha}$-silyloxyketone 11. To a stirred solution of enone $\mathbf{1 1}$ (2 mg, 4 $\mu \mathrm{mol})$ in $\mathrm{MeOH}(600 \mu \mathrm{L})$ ain an ice bath was added batches of $\mathrm{NaBH}_{4}(2 \mathrm{mg}, 50 \mu \mathrm{mol}$, 25 equiv) every $10 \mathrm{~min}$ for $1 \mathrm{hr}(\sim 150$ equiv total) while warming to room temperature. Aqueous $\mathrm{HCl}(3 \mathrm{~N}, 1 \mathrm{~mL})$ was added to the reaction and stirred for an additional $5 \mathrm{~min}$ before extracting with $\mathrm{Et}_{2} \mathrm{O}$. The organic phase was washed with sat'd $\mathrm{NaHCO}_{3}$ and brine, and concentrated in vacuo. The resulting oil was azeotroped with $\mathrm{MeOH}(3 \times 10$ $\mathrm{mL})$ and chromatographed on silica gel (6:1 hexanes:EtOAc eluent) to give alcohol $\mathbf{1 3}$ $(1.8 \mathrm{mg}, 90 \%)$ as a colorless oil.

\section{Deprotection of TMS ether 13.}

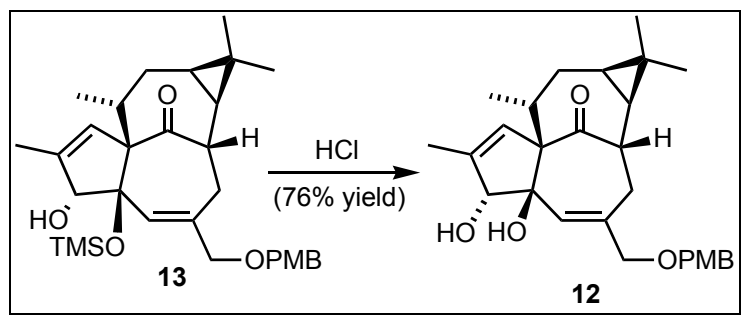

Deprotection of TMS ether 13. A stirred solution of TMS ether $\mathbf{1 3}(1.8 \mathrm{mg}, 4$ $\mu \mathrm{mol})$ and $\mathrm{HCl}(1 \mathrm{M}, 500 \mu \mathrm{L})$ in $\mathrm{THF}(500 \mu \mathrm{L})$ was allowed to stir at room temperature for $20 \mathrm{hrs}$ before aqueous $\mathrm{NaHCO}_{3}$ was added, and the solution was extracted with EtOAc. The organic layer was washed with brine, dried over $\mathrm{MgSO}_{4}$, and the solvent was removed in vacuo. Silica gel chromatography (3:1 hexane:EtOAc eluent) afforded diol $12(1.2 \mathrm{mg}, 76 \%)$ as a colorless oil. 


\section{Preparation of epoxide 14.}

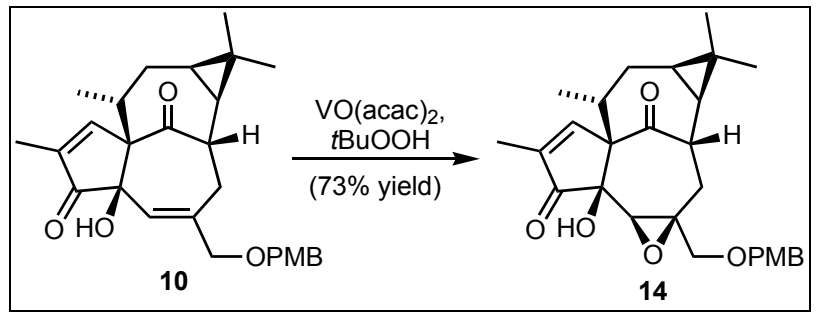

Epoxide 14. A stirred solution of diene $10(20 \mathrm{mg}, 44 \mu \mathrm{mol})$ and $\mathrm{VO}(\mathrm{acac})_{2}(2$ mg, $7 \mu \mathrm{mol}, 15 \mathrm{~mol} \%)$ in benzene $(4 \mathrm{ml})$ was cooled to $10^{\circ} \mathrm{C}$ before the addition of $t$ butyl hydroperoxide (5-6 M in decane, $50 \mu \mathrm{L}, \sim 250 \mu \mathrm{mol}, 6$ equiv). Upon addition, the solution turns from green to maroon. The reaction was allowed to warm to room temperature and stir an additional $10 \mathrm{hrs}$, at which point the solution was introduced directly to silica gel and chromatographed (11:1 hexane:EtOAc eluent) to give epoxide $14(15 \mathrm{mg}, 73 \%)$ as a white solid.

Epoxide 14: m.p. $141^{\circ} \mathrm{C}$; FTIR (thin film/NaCl) 3430 (br w), 2927 (m), 1717 (s), $1514(\mathrm{~m}), 1248$ (m), 1035 (w), 734 (w) $\mathrm{cm}^{-1} ;{ }^{1} \mathrm{H}$ NMR (400 MHz, $\left.\mathrm{CDCl}_{3}\right) \delta 7.68$ (d, J= $1 \mathrm{~Hz}, 1 \mathrm{H}), 7.19$ (br d, $J=8 \mathrm{~Hz}, 2 \mathrm{H}), 6.86$ (br d, $J=8 \mathrm{~Hz}, 2 \mathrm{H}), 4.38(\mathrm{~d}, J=11.5 \mathrm{~Hz}, 1 \mathrm{H})$, $4.34(\mathrm{~d}, J=11.5 \mathrm{~Hz}, 1 \mathrm{H}), 3.80(\mathrm{~s}, 3 \mathrm{H}), 3.37(\mathrm{~s}, 1 \mathrm{H}), 3.33(\mathrm{~d}, J=11.5 \mathrm{~Hz}, 1 \mathrm{H}), 3.21(\mathrm{~d}, J$ $=11.5 \mathrm{~Hz}, 1 \mathrm{H}), 2.78(\mathrm{~s}, 1 \mathrm{H}), 2.63(\mathrm{ddd}, J=3,11,14 \mathrm{~Hz}, 1 \mathrm{H}), 2.34(\mathrm{t}, J=14 \mathrm{~Hz}, 1 \mathrm{H})$, 2.34-2.26 (m, 1H), 2.16-2.13 (m, 1H), $2.07(\mathrm{dd}, J=3,14 \mathrm{~Hz}, 1 \mathrm{H}), 1.86(\mathrm{~d}, J=1 \mathrm{~Hz}, 3 \mathrm{H})$, $1.75(\mathrm{ddd}, J=4.5,6,16 \mathrm{~Hz}, 1 \mathrm{H}), 1.09$ (s, 3H), 1.03 (s, 3H), 1.02 (d, $J=8 \mathrm{~Hz}, 3 \mathrm{H}), 0.72-$ $0.66(\mathrm{~m}, 1 \mathrm{H}), 0.60(\mathrm{dd}, J=8,11 \mathrm{~Hz}, 1 \mathrm{H}) ;{ }^{13} \mathrm{C} \mathrm{NMR}\left(100 \mathrm{MHz}, \mathrm{CDCl}_{3}\right) \delta 208.4,206.4$, $159.5,158.8,138.4,129.8,129.6,114.0,78.5,73.1,73.0,71.3,69.0,65.6,55.5,41.3$, 40.0, 30.2, 29.3, 28.8, 24.0, 23.7, 23.0, 17.3, 15.2, 11.1; LRMS (EI) $m / z 489.3\left(\mathrm{~m}+\mathrm{Na}^{+}\right)$. 


\section{Preparation of TMS ether 16.}

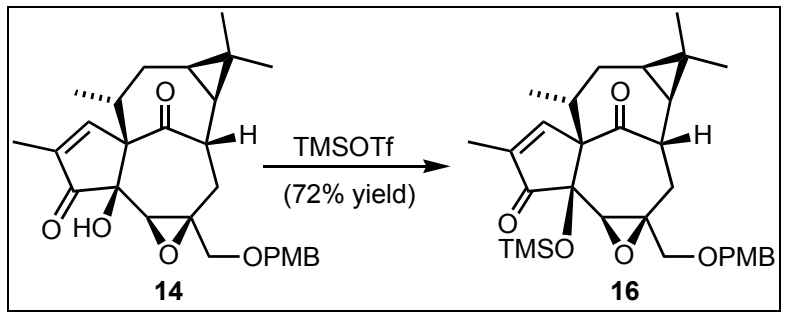

TMS ether 16. To a stirred solution of alcohol $14(13 \mathrm{mg}, 28 \mu \mathrm{mol})$ in $\mathrm{CH}_{2} \mathrm{Cl}_{2}$ $(1.5 \mathrm{~mL})$ was added triethylamine $(750 \mu \mathrm{L}, 5 \mathrm{mmol}, 175$ equiv). The solution was cooled to $-10^{\circ} \mathrm{C}$ in a $\mathrm{MeOH} / \mathrm{NaCl} /$ ice bath and TMSOTf $(30 \mu \mathrm{L}, 150 \mu \mathrm{mol}, 5$ equiv) was added at once via syringe. The reaction was allowed to warm to $-5^{\circ} \mathrm{C}$ over $1 \mathrm{hr}$, at which time the reaction was quenched with aqueous $\mathrm{NaHCO}_{3}$ and extracted with $\mathrm{CH}_{2} \mathrm{Cl}_{2}$. The organic phase was washed with brine, dried over $\mathrm{MgSO}_{4}$, and filtered. Following concentration in vacuo, flash chromatography on silica gel (15:1 hexane:EtOAc eluent) afforded TMS ether $\mathbf{1 6}(11 \mathrm{mg}, 72 \%)$ as a colorless oil.

TMS ether 16: FTIR (thin film/NaCl) 3430 (br), 2927 (m), 1717 (s), 1514 (m), 1248 (m), 1035 (w), $734(\mathrm{w}) \mathrm{cm}^{-1} ;{ }^{1} \mathrm{H}$ NMR (500 MHz, $\left.\mathrm{CDCl}_{3}\right) \delta 7.64$ (d, $\left.J=1 \mathrm{~Hz}, 1 \mathrm{H}\right)$, $7.20(\mathrm{~d}, J=8.5 \mathrm{~Hz}, 2 \mathrm{H}), 7.85(\mathrm{~d}, J=8.5 \mathrm{~Hz}, 2 \mathrm{H}), 4.39(\mathrm{~d}, J=11.5,1 \mathrm{H}), 4.34(\mathrm{~d}, J=$ 11.5, 1H), $3.80(\mathrm{~s}, 3 \mathrm{H}), 3.30(\mathrm{~d}, J=11.5 \mathrm{~Hz}, 1 \mathrm{H}), 3.21(\mathrm{~d}, J=11.5,1 \mathrm{H}), 2.73(\mathrm{~s}, 1 \mathrm{H})$, $2.64(\mathrm{ddd}, J=3,12,14 \mathrm{~Hz}), 2.30(\mathrm{t}, J=14 \mathrm{~Hz}, 1 \mathrm{H}), 2.28-2.20(\mathrm{~m}, 1 \mathrm{H}), 2.03(\mathrm{dd}, J=3$, $14 \mathrm{~Hz}, 1 \mathrm{H}), 1.82(\mathrm{~d}, J=1 \mathrm{~Hz}, 3 \mathrm{H}), 1.72(\mathrm{ddd}, J=4,6,16 \mathrm{~Hz}, 1 \mathrm{H}), 1.07$ (s, 3H), 1.02 (s, $3 \mathrm{H}), 1.01(\mathrm{~d}, J=7.5 \mathrm{~Hz}, 3 \mathrm{H}), 0.68-0.63(\mathrm{~m}, 1 \mathrm{H}), 0.59(\mathrm{dd}, J=8.5,11 \mathrm{~Hz}, 1 \mathrm{H}), 0.27(\mathrm{~s}$, $9 \mathrm{H}) ;{ }^{13} \mathrm{C}$ NMR $\left(125 \mathrm{MHz}, \mathrm{CDCl}_{3}\right) \delta 209.4,206.3,159.5,158.7,137.9,136.0,114.0$, $81.9,73.6,73.1,72.0,68.1,66.0,55.5,41.2,40.2,30.5,30.0,29.5,28.8,24.1,23.0,17.2$, 15.2, 11.0, 2.2; LRMS (EI) $m / z 561.4\left(\mathrm{~m}+\mathrm{Na}^{+}\right)$. 


\section{Preparation of acetonide 17.}

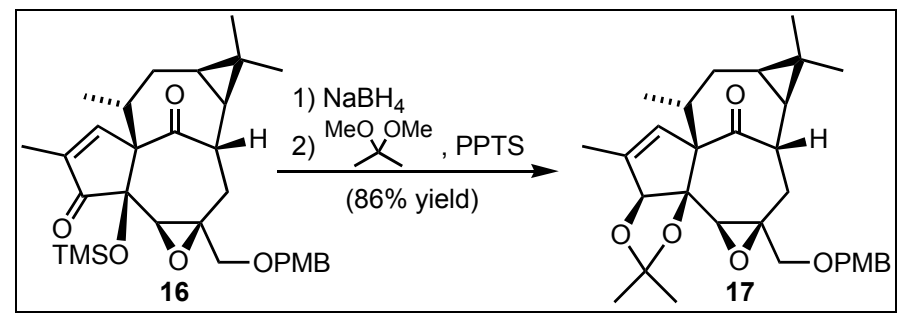

Acetonide 17. To a stirred solution of enone $16(48 \mathrm{mg}, 89 \mu \mathrm{mol})$ in $\mathrm{MeOH}(5$ $\mathrm{mL})$ at room temperature was added batches of $\mathrm{NaBH}_{4}(5 \mathrm{mg}, 130 \mu \mathrm{mol}, 1.5$ equiv) every 20 min for 5 hrs ( 20 equiv total). Aqueous $\mathrm{HCl}(1 \mathrm{~N}, 5 \mathrm{~mL}, \sim 50$ equiv) was added to the reaction and stirred for an additional 20 min before extracting with EtOAc. The organic phase was washed with sat'd $\mathrm{NaHCO}_{3}$ and brine, and concentrated in vacuo. The resulting oil was azeotroped with $\mathrm{MeOH}(3 \times 50 \mathrm{~mL})$ to give diol 16' as a crude oil, which was carried forward without further purification.

Crude diol 16' was dissolved in $\mathrm{CH}_{2} \mathrm{Cl}_{2}(7 \mathrm{~mL})$. 2,2-Dimethoxypropane (3 mL, 25 mmol, 275 equiv) and PPTS $(\sim 0.2 \mathrm{mg})$ were added to the solution, which was immediately heated to reflux. After $30 \mathrm{~min}$, the reaction was cooled to room temperature and diluted with $\mathrm{CH}_{2} \mathrm{Cl}_{2}$. The organic phase was washed with sat'd $\mathrm{NaHCO}_{3}$ and brine, dried over $\mathrm{MgSO}_{4}$, and concentrated in vacuo to give a crude oil, which after silica gel chromatography (12:1 hexane:EtOAc eluent), afforded acetonide 17 (39 mg, 86\% over two steps) as a colorless oil.

Acetonide 17: FTIR (thin film/NaCl) 2925 (m), 2866 (m), 1721 (s), 1613 (w), 1514 (s), 1249 (s), 1036 (s) cm ${ }^{-1} ;{ }^{1} \mathrm{H}$ NMR (500 MHz, $\left.\mathrm{CDCl}_{3}\right) \delta 7.23$ (d, J=8.5 Hz, 2H), $6.86(\mathrm{~d}, J=8.5 \mathrm{~Hz}, 2 \mathrm{H}), 5.87(\mathrm{~d}, 1.5 \mathrm{~Hz}, 1 \mathrm{H}), 4.61(\mathrm{~s}, 1 \mathrm{H}), 4.41(\mathrm{~s}, 2 \mathrm{H}), 3.80(\mathrm{~s}, 3 \mathrm{H})$, $3.39(\mathrm{~d}, J=11.5 \mathrm{~Hz}, 1 \mathrm{H}), 3.28(\mathrm{~d}, J=11.5 \mathrm{~Hz}, 1 \mathrm{H}), 2.94(\mathrm{~s}, 1 \mathrm{H}), 2.60(\mathrm{ddd}, J=3,11,14$ 
Hz, 1H), 2.34-2.29 (m, 1H), $2.31(\mathrm{t}, J=14 \mathrm{~Hz}, 1 \mathrm{H}), 2.19(\mathrm{ddd}, J=2.5,8,16,1 \mathrm{H}), 2.02$ (dd, $J=2.5,14 \mathrm{~Hz}), 1.85(\mathrm{~d}, J=1 \mathrm{~Hz}, 3 \mathrm{H}), 1.66(\mathrm{dt}, J=6,6,16 \mathrm{~Hz}, 1 \mathrm{H}), 1.54(\mathrm{~s}, 3 \mathrm{H})$, $1.53(\mathrm{~s}, 3 \mathrm{H}), 1.10(\mathrm{~s}, 3 \mathrm{H}), 1.00(\mathrm{~s}, 3 \mathrm{H}), 0.95(\mathrm{~d}, J=8 \mathrm{~Hz}, 3 \mathrm{H}), 0.64-0.54(\mathrm{~m}, 2 \mathrm{H}),{ }^{13} \mathrm{C}$ NMR (125 MHz, $\left.\mathrm{CDCl}_{3}\right) \delta$ 211.1, 159.5, 135.3, 132.5, 130.2, 129.6, 114.7, 114.0, 95.0, $90.6,76.3,73.5,73.0,68.0,66.7,55.5,41.1,40.1,29.9,29.9,29.3,28.8,26.4,26.3,23.8$, 23.1, 17.1, 15.7, 15.3; LRMS (EI) $m / z 531.4\left(\mathrm{~m}+\mathrm{Na}^{+}\right)$.

\section{Preparation of alcohol 18.}

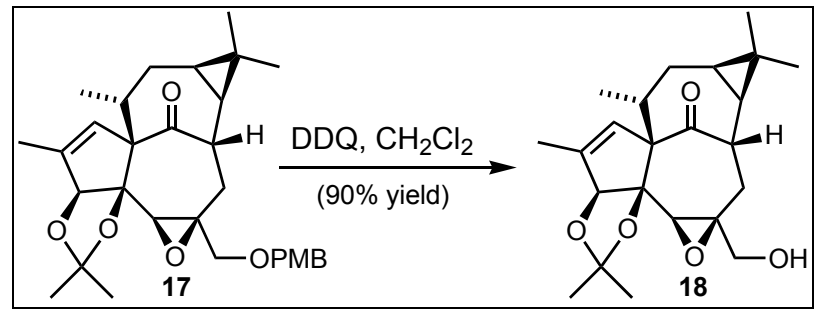

Alcohol 18. A solution of PMB ether 17 (35 mg, $69 \mu \mathrm{mol})$ and 2,3-dichloro-5,6dicyano-1,4-benzoquinone ( $35 \mathrm{mg}, 150 \mu \mathrm{mol}, 2.2$ equiv) in $\mathrm{CH}_{2} \mathrm{Cl}_{2}(2 \mathrm{~mL})$ in an open flask was was allowed to stir at room temperature for 18 hours. The reaction was poured into sat'd $\mathrm{NaHCO}_{3}$ and extracted with $\mathrm{CH}_{2} \mathrm{Cl}_{2}$. The organic phase was washed with sat'd $\mathrm{NaHCO}_{3}$ and brine, dried over $\mathrm{MgSO}_{4}$, and concentrated in vacuo. The crude residue was chromatographed on silica gel $(6: 1 \rightarrow 1: 1$ hexanes:EtOAc eluent) to afford alcohol 18 (24 $\mathrm{mg}, 90 \%)$ as a colorless oil.

Alcohol 18: FTIR (thin film/NaCl) 3483 (br), 2922 (s), 1721 (s), 1381 (m), 1206

(s), $1037(\mathrm{~m}) \mathrm{cm}^{-1} ;{ }^{1} \mathrm{H}$ NMR $\left(400 \mathrm{MHz}, \mathrm{CDCl}_{3}, 40^{\circ} \mathrm{C}\right) \delta 5.88(\mathrm{br} \mathrm{s}, 1 \mathrm{H}), 4.65(\mathrm{~s}, 1 \mathrm{H})$, 3.57 (d, $J=12 \mathrm{~Hz}, 1 \mathrm{H}), 3.50$ (d, $J=12 \mathrm{~Hz}, 1 \mathrm{H}), 3.12$ (s, 1H), 2.64 (ddd, $J=3,11,14$, Hz, 1H), 2.33 (m, 1H), 2.21 (ddd, $J=2,10,14 \mathrm{~Hz}, 1 \mathrm{H}), 2.15$ (t, $J=14 \mathrm{~Hz}, 1 \mathrm{H}), 1.96$ (dd, $J=3,14 \mathrm{~Hz}, 1 \mathrm{H}), 1.86(\mathrm{br} \mathrm{s}, 3 \mathrm{H}), 1.55$ (s, 3H), 1.53 (s, 3H), 1.11 (s, 3H), 1.01 (s, 3H), 
$0.96(\mathrm{~d}, J=7 \mathrm{~Hz}, 3 \mathrm{H}), 0.54-0.46(\mathrm{~m}, 2 \mathrm{H}) ;{ }^{13} \mathrm{C} \mathrm{NMR}\left(100 \mathrm{MHz}, \mathrm{CDCl}_{3}, 40^{\circ} \mathrm{C}\right) \delta 210.8$, $135.4,132.7,114.8,95.1,90.5,76.3,67.2,66.7,64.9,41.2,40.3,29.9,29.8,28.8,26.4$, 26.3, 23.8, 23.2, 23.2, 17.1, 15.7, 15.2; LRMS (EI) $m / z 411.3\left(\mathrm{~m}+\mathrm{Na}^{+}\right)$.

\section{Preparation of mesylate 19.}

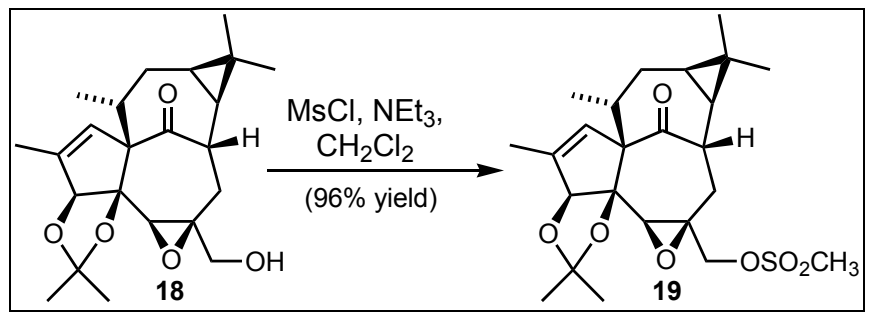

Mesylate 19. A stirred solution of alcohol $18(24 \mathrm{mg}, 62 \mu \mathrm{mol})$ and triethylamine ( $800 \mu \mathrm{L}, 5.7 \mathrm{mmol}, 100$ equiv) in $\mathrm{CH}_{2} \mathrm{Cl}_{2}(1 \mathrm{~mL})$ was cooled to $-78^{\circ} \mathrm{C}$ in an acetone $/ \mathrm{CO}_{2}$ bath. Methanesulfonyl chloride ( $40 \mu \mathrm{L}, 525 \mu$ mol, 8.5 equiv) was added dropwise via syringe, and the solution was allowed to react for $45 \mathrm{~min}$ at $-78^{\circ} \mathrm{C}$, at which time sat'd $\mathrm{NaHCO}_{3}$ was added, the solution was allowed to warm to room temperature and extracted with $\mathrm{CH}_{2} \mathrm{Cl}_{2}$. The organic phase was washed with brine, dried over $\mathrm{MgSO}_{4}$, and concentrated in vacuo. The residue was subjected to silica gel chromatography (5:1 hexanes:EtOAc eluent) to provide mesylate $19(28 \mathrm{mg}, 96 \%)$ as a colorless oil.

Mesylate 19: FTIR (thin film/NaCl) 2926 (m), 1721 (s), 1359 (s), 1177 (s), 956 (m) $\mathrm{cm}^{-1} ;{ }^{1} \mathrm{H}$ NMR $\left(400 \mathrm{MHz}, \mathrm{CDCl}_{3}\right) \delta 5.87(\mathrm{~d}, J=1 \mathrm{~Hz}, 1 \mathrm{H}), 4.65(\mathrm{~s}, 1 \mathrm{H}), 4.20(\mathrm{~d}$, $11.5 \mathrm{~Hz}, 1 \mathrm{H}), 3.97$ (d, $J=11.5 \mathrm{~Hz}, 1 \mathrm{H}), 3.05(\mathrm{~s}, 3 \mathrm{H}), 2.99$ (s, 1H), 2.56 (ddd, $J=3,11.5$, $14 \mathrm{~Hz}, 1 \mathrm{H}), 2.31(\mathrm{t}, J=13.5 \mathrm{~Hz}, 1 \mathrm{H}), 2.33-2.28(\mathrm{~m}, 1 \mathrm{H}), 2.18$ (ddd, $J=3,11.5,14 \mathrm{~Hz}$, $1 \mathrm{H}), 2.07(\mathrm{dd}, J=2.5,14 \mathrm{~Hz}, 1 \mathrm{H}), 1.87(\mathrm{~d}, J=1 \mathrm{~Hz}, 3 \mathrm{H}), 1.67(\mathrm{dt}, J=6.6,15.5,1 \mathrm{H})$, $1.54(\mathrm{~s}, 3 \mathrm{H}), 1.52(\mathrm{~s}, 3 \mathrm{H}), 1.09(\mathrm{~s}, 3 \mathrm{H}), 1.01(\mathrm{~s}, 3 \mathrm{H}), 0.95(\mathrm{~d}, J=7 \mathrm{~Hz}, 3 \mathrm{H}), 0.64(\mathrm{dt}, J=$ 
6, 9, $9 \mathrm{~Hz}, 1 \mathrm{H}), 0.55(\mathrm{dd}, J=8.5,11 \mathrm{~Hz}, 1 \mathrm{H}) ;{ }^{13} \mathrm{C} \mathrm{NMR}\left(100 \mathrm{MHz}, \mathrm{CDCl}_{3}\right) \delta 210.5$, $135.3,132.5,115.0,94.9,20.3,76.3,72.6,68.2,64.6,41.0,40.3,38.2,29.6,29.0,28.7$, 26.3, 26.1, 23.5, 23.3, 23.0, 17.0, 15.8, 15.2; LRMS (EI) $m / z 489.3\left(\mathrm{~m}+\mathrm{Na}^{+}\right)$.

\section{Preparation of sulfide 20.}

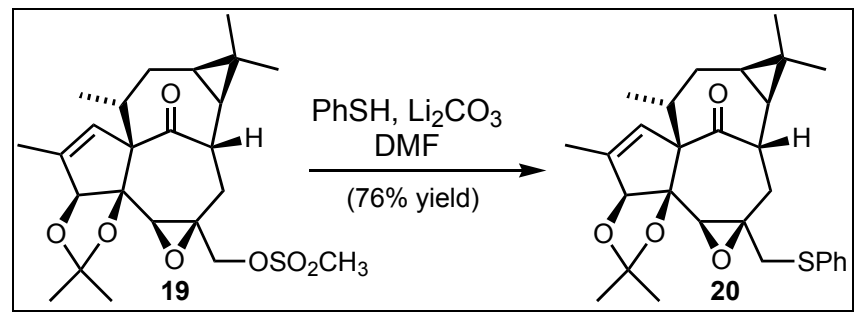

Sulfide 20. To a stirred solution of mesylate 19 (39 mg, $84 \mu \mathrm{mol})$ in DMF (600 $\mu \mathrm{L})$ was added $\mathrm{Li}_{2} \mathrm{CO}_{3}(130 \mathrm{mg}, 1.7 \mathrm{mmol}, 20$ equiv). The suspension was warmed to $55^{\circ} \mathrm{C}$ for $10 \mathrm{~min}$, at which point thiophenol $(75 \mu \mathrm{L}, 730 \mu \mathrm{mol}, 9$ equiv) was added via syringe. The mixture was allowed to react at $55^{\circ} \mathrm{C}$ for $5 \mathrm{hrs}$, at which point the reaction was partitioned between ether and water. The organic layer was washed with water and brine, dried over $\mathrm{MgSO}_{4}$, and concentrated in vacuo. The residual oil was chromatographed (40:1 $\rightarrow 20: 1$ hexanes:EtOAc eluent) to afford sulfide 20 (31 mg, 76\%) as a pale yellow oil.

Sulfide 20: FTIR (thin film/NaCl) 2921 (s), 1722 (s), 1381 (m), 1206 (m), 738 (m) $\mathrm{cm}^{-1} ;{ }^{1} \mathrm{H}$ NMR $\left(500 \mathrm{MHz}, \mathrm{CDCl}_{3}\right) \delta 7.34(\mathrm{~d}, J=7 \mathrm{~Hz}, 2 \mathrm{H}), 7.24(\mathrm{~m}, 2 \mathrm{H}), 7.20(\mathrm{t}, J=$ $7 \mathrm{~Hz}), 5.80(\mathrm{~s}, 1 \mathrm{H}), 4.24(\mathrm{~s}, 1 \mathrm{H}), 2.99(\mathrm{~d}, J=14 \mathrm{~Hz}, 1 \mathrm{H}), 2.89(\mathrm{~d}, J=14 \mathrm{~Hz}, 1 \mathrm{H}), 2.72(\mathrm{~s}$, 1H), 2.62-2.52 (m, 2H), 2.28-2.25 (m, 1H), $2.15(\mathrm{dd}, J=8,14 \mathrm{~Hz}, 1 \mathrm{H}), 2.03(\mathrm{~d}, J=11$ Hz, 1H), 1.76 (s, 3H), 1.65 (dt, $J=6,6,15 \mathrm{~Hz}, 1 \mathrm{H}), 1.49$ (s, 3H), 1.46 (s, 3H), 1.09 (s, $3 \mathrm{H}), 1.00(\mathrm{~s}, 3 \mathrm{H}), 0.94(\mathrm{~d}, J=7 \mathrm{~Hz}, 3 \mathrm{H}), 0.63-0.56(\mathrm{~m}, 2 \mathrm{H}) ;{ }^{13} \mathrm{C} \mathrm{NMR}(125 \mathrm{MHz}$, $\left.\mathrm{CDCl}_{3}\right) \delta 210.4,135.5,135.2,132.2,131.2,129.2,126.9,114.5,94.8,90.7,76.3,70.7$, 
$66.6,43.3,41.2,40.0,30.9,30.0,28.8,26.5,26.3,23.9,23.1,23.1,17.1,15.8,15.3$;

LRMS (EI) $m / z 503.3\left(\mathrm{~m}+\mathrm{Na}^{+}\right)$.

\section{Preparation of sulfone 21.}

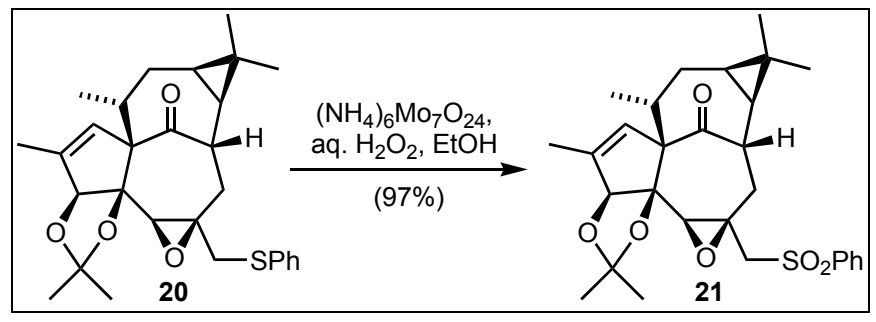

Sulfone 21. To a stirred solution of sulfide $20(30 \mathrm{mg}, 62 \mu \mathrm{mol})$ in ethanol (95\%,

$2 \mathrm{ml}$ ) was added ammonium molybdate tetrahydrate (18 mg, $15 \mu \mathrm{mol}, 24 \mathrm{~mol} \%)$. The resulting suspension was stirred at room temperature for 10 min, and $\mathrm{H}_{2} \mathrm{O}_{2}(30 \%, 350 \mu \mathrm{L}$, $3.1 \mathrm{mmol}, 50$ equiv) was added dropwise. The reaction was allowed to proceed at room temperature for $6 \mathrm{hrs}$, at which point the solution was cooled in an ice bath and aqueous $\mathrm{Na}_{2} \mathrm{SO}_{3}$ was added. After warming to room temperature over 15 minutes, the reaction was partitioned between $\mathrm{H}_{2} \mathrm{O}$ and EtOAc, the organic layer was washed with water and brine, dried over $\mathrm{MgSO}_{4}$, and concentrated in vacuo. Flash chromatography (3:1 hexanes:EtOAc eluent) affored sulfone 21 (31 mg, 97\%) as a colorless oil.

Sulfone 21: FTIR (thin film/NaCl) 2943 (m), 1717 (s), 1312 (s), 1206 (m), 1161 (s), $1087(\mathrm{~m}) \mathrm{cm}^{-1} ;{ }^{1} \mathrm{H}$ NMR $\left(400 \mathrm{MHz}, \mathrm{CDCl}_{3}\right) \delta 7.87(\mathrm{~d}, J=7.5 \mathrm{~Hz}, 2 \mathrm{H}), 7.66(\mathrm{t}, J=$ $7.5 \mathrm{~Hz}, 1 \mathrm{H}), 7.55$ (t, $J=7.5 \mathrm{~Hz}, 2 \mathrm{H}), 5.77(\mathrm{~d}, J=1 \mathrm{~Hz}, 1 \mathrm{H}), 4.23(\mathrm{~s}, 1 \mathrm{H}), 3.41$ (d, $J=$ $14.5 \mathrm{~Hz}, 1 \mathrm{H}), 3.07(\mathrm{~d}, J=14.5 \mathrm{~Hz}, 1 \mathrm{H}), 2.68(\mathrm{t}, J=13.5 \mathrm{~Hz}, 1 \mathrm{H}), 2.63(\mathrm{~s}, 1 \mathrm{H}), 2.55-2.47$ (m, 1H), 2.30-2.22 (m, 1H), $2.13(\mathrm{dd}, J=1.5,13.5 \mathrm{~Hz}, 1 \mathrm{H}), 2.09-2.05(\mathrm{~m}, 1 \mathrm{H}), 1.80(\mathrm{~d}, J$ $=1 \mathrm{~Hz}, 3 \mathrm{H}), 1.65(\mathrm{dt}, J=6,6,15.5 \mathrm{~Hz}), 1.47(\mathrm{~s}, 3 \mathrm{H}), 1.42(\mathrm{~s}, 3 \mathrm{H}), 1.07(\mathrm{~s}, 3 \mathrm{H}), 0.98(\mathrm{~s}$, $3 \mathrm{H}), 0.93(\mathrm{~d}, J=7 \mathrm{~Hz}, 3 \mathrm{H}), 0.61-0.55(\mathrm{~m}, 2 \mathrm{H}) ;{ }^{13} \mathrm{C} \mathrm{NMR}\left(100 \mathrm{MHz}, \mathrm{CDCl}_{3}\right) \delta 209.9$, 
139.6, 135.5, 134.0, 131.6, 129.6, 128.6, 114.6, 94.5, 90.6, 76.2, 70.7, 64.7, 62.7, 40.9,

39.7, 31.6, 30.2, 28.7, 26.8, 26.4, 23.8, 23.1, 23.0, 17.3, 15.7, 15.2; LRMS (EI) $m / z 535.4$ $\left(\mathrm{m}+\mathrm{Na}^{+}\right)$.

\section{Isomerization of epoxide 21.}

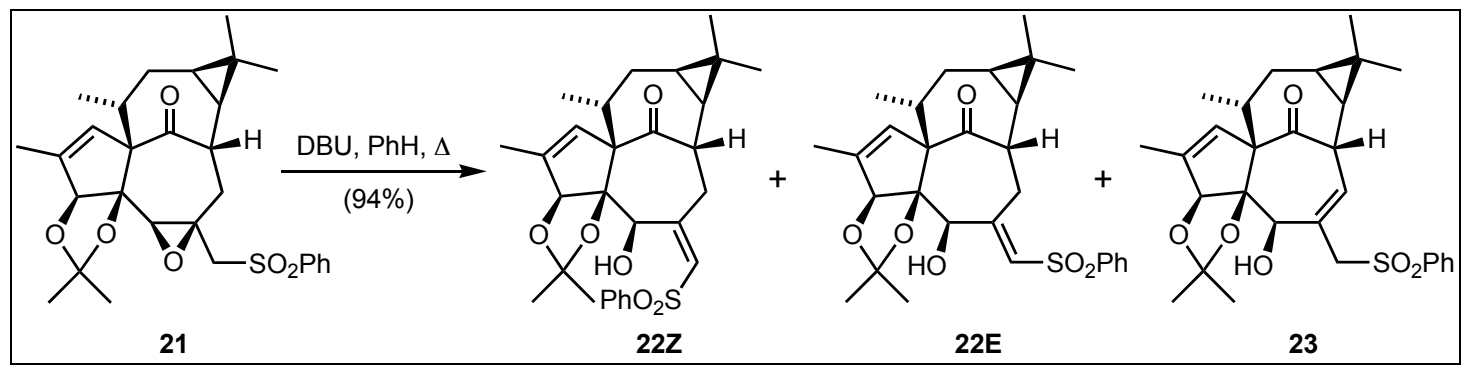

Isomerization of epoxide 21. To a stirred solution of epoxide 21 (15 mg, 29 $\mu \mathrm{mol})$ in benzene $(2.5 \mathrm{~mL})$ was added 1,8-Diazabicyclo[5.4.0]undec-7-ene (10 $\mu \mathrm{L}, 67$ $\mu \mathrm{mol}, 2.2$ equiv) at room tempertature. The reaction was refluxed under an atmosphere of $\mathrm{N}_{2}$ for 5 hrs, at which time the reaction was cooled to room temperature and introduced directly to silica gel. Elution (50:1 $\rightarrow 40: 1$ benzene:EtOAc) afforded Z-vinyl sulfone $22 \mathrm{Z}$ (white solid, $1 \mathrm{mg}, 7 \%$ ), E-vinyl sulfone $22 \mathrm{E}$ (colorless oil, $6 \mathrm{mg}, 40 \%$ ), and allylic sulfone $\mathbf{2 3}$ (colorless needles, $7 \mathrm{mg}, \mathbf{4 7 \%}$ ). Recrystallization of allylic sulfone $\mathbf{2 3}$ with pentane afforded colorless needles, upon which X-Ray diffraction analysis was performed (see cif file).

Vinyl sufones $22 \mathrm{E}$ and $\mathbf{2 2 Z}$ (30 $\mathrm{mg}, 55 \mu \mathrm{mol}$ ) were combined and dissolved in benzene $(3 \mathrm{~mL}) .1,8$-Diazabicyclo[5.4.0]undec-7-ene (10 $\mu \mathrm{L}, 67 \mu \mathrm{mol}, 1.2$ equiv) was added and the reaction was refluxed for $4 \mathrm{hrs,}$, at which point the solution was cooled and introduced to silica. Elution (50:1 $\rightarrow 40: 1$ benzene:EtOAc) afforded allylic sulfone 23 (14 $\mathrm{mg}, 47 \%)$ and a mixture of vinyl sulfones $22(15 \mathrm{mg}, 50 \%)$. 
Z-Vinyl sulfone 22Z: m.p.: 194-198 (decomp.); FTIR (thin film/NaCl) 3550 (br), 2925 (m), 1721 (m), 1306 (m), 1238(m), 1151 (s), 738 (s) cm ${ }^{-1} ;{ }^{1} \mathrm{H}$ NMR (500 MHz, $\left.\mathrm{CDCl}_{3}\right) \delta 7.88(\mathrm{~d}, J=7.5 \mathrm{~Hz}, 2 \mathrm{H}), 7.62(\mathrm{t}, J=7.5 \mathrm{~Hz}, 1 \mathrm{H}), 7.54(\mathrm{t}, J=7.5 \mathrm{~Hz}, 2 \mathrm{H}), 6.26$ (d, $J=1 \mathrm{~Hz}, 1 \mathrm{H}), 5.91(\mathrm{~s}, 1 \mathrm{H}), 5.37(\mathrm{~s}, 1 \mathrm{H}), 4.90(\mathrm{~s}, 1 \mathrm{H}), 4.17$ (ddd, $J=5.5,9,14 \mathrm{~Hz}$, $1 \mathrm{H}), 3.38(\mathrm{~s}, 1 \mathrm{H}), 2.74-2.68(\mathrm{~m}, 2 \mathrm{H}), 2.38(\mathrm{t}, J=14 \mathrm{~Hz}, 1 \mathrm{H}), 1.94(\mathrm{br} \mathrm{d}, J=16 \mathrm{~Hz}, 1 \mathrm{H})$, $1.82(\mathrm{~s}, 3 \mathrm{H}), 1.81-1.74(\mathrm{~m}, 1 \mathrm{H}), 1.60(\mathrm{~s}, 3 \mathrm{H}), 1.42(\mathrm{~s}, 3 \mathrm{H}), 1.21(\mathrm{~s}, 3 \mathrm{H}), 0.98(\mathrm{~s}, 3 \mathrm{H}), 0.98$ $(\mathrm{d}, J=7 \mathrm{~Hz}, 3 \mathrm{H}), 0.76(\mathrm{t}, J=10 \mathrm{~Hz}, 1 \mathrm{H}), 0.63(\mathrm{ddd}, J=2.5,6.5,9 \mathrm{~Hz}, 1 \mathrm{H}) ;{ }^{13} \mathrm{C} \mathrm{NMR}$ $\left(125 \mathrm{MHz}, \mathrm{CDCl}_{3}\right) \delta 212.8,151.4,141.3,140.4,133.8,131.7,129.5,127.6,125.5,113.2$, $94.8,89.3,75.5,72.4,40.2,36.5,35.7,33.5,29.7,29.1,28.2,25.4,25.2,22.1,19.5,15.4$, 14.2; LRMS (EI) $m / z 535.3\left(\mathrm{~m}+\mathrm{Na}^{+}\right)$.

E-Vinyl sulfone 22E: FTIR (thin film/NaCl) 3460 (br), 2924 (m), 1720 (s), 1447 (m), 1382 (m), 1150 (s), 1084 (s), 735 (s) cm ${ }^{-1} ;{ }^{1} \mathrm{H}$ NMR (500 MHz, $\left.\mathrm{CDCl}_{3}\right) \delta 7.91$ (d, $J$ $=7 \mathrm{~Hz}, 2 \mathrm{H}), 7.62(\mathrm{br} \mathrm{t}, J=7 \mathrm{~Hz}, 1 \mathrm{H}), 7.55(\mathrm{t}, J=7 \mathrm{~Hz}, 2 \mathrm{H}), 6.34(\mathrm{~s}, 1 \mathrm{H}), 5.45(\mathrm{~s}, 1 \mathrm{H})$, 4.40 (s, 1H), 4.14 (s, 1H), 3.83 (ddd, $J=4.5,9,14 \mathrm{~Hz}, 1 \mathrm{H}), 3.52$ (t, $J=14 \mathrm{~Hz}, 1 \mathrm{H}), 3.10$ (s, $1 \mathrm{H}), 2.70$ (ddd, $J=1.5,4.5,15 \mathrm{~Hz}, 1 \mathrm{H}), 2.65-2.60(\mathrm{~m}, 1 \mathrm{H}), 1.95$ (dt, $J=3,3,16 \mathrm{~Hz}$, 1H), 1.80-1.73 (m, 1H), $1.73(\mathrm{~s}, 3 \mathrm{H}), 1.45(\mathrm{~s}, 3 \mathrm{H}), 1.37$ (s, 3H), $1.18(\mathrm{~s}, 3 \mathrm{H}), 1.01(\mathrm{~s}, 3 \mathrm{H})$, $0.95(\mathrm{~d}, J=6.5 \mathrm{~Hz}, 3 \mathrm{H}), 0.85(\mathrm{dd}, J=9,10.5 \mathrm{~Hz}, 1 \mathrm{H}), 0.63(\mathrm{ddd}, J=3,6.5,9.5 \mathrm{~Hz}, 1 \mathrm{H})$; ${ }^{13} \mathrm{C}$ NMR $\left(125 \mathrm{MHz}, \mathrm{CDCl}_{3}\right) \delta 212.5,153.9,141.5,138.8,133.7,131.3,129.4,127.5$, $127.4,113.5,95.2,89.1,81.6,74.9,40.5,36.5,33.3,29.3,29.1,28.2,27.9,25.3,25.0$, 22.3, 19.4, 15.4, 14.2; LRMS (EI) $m / z 535.5\left(\mathrm{~m}+\mathrm{Na}^{+}\right)$.

Allylic sulfone 23: m.p.: $185-186^{\circ} \mathrm{C}$ (decomp.); FTIR (thin film/ $/ \mathrm{NaCl}$ ) 3462 (br), 2936 (m), 1725 (s), 1447 (m), 1307 (s), 1144 (s), 731 (s) (m) cm ${ }^{-1} ;{ }^{1} \mathrm{H}$ NMR (400 MHz, $\left.\mathrm{CDCl}_{3}\right) \delta 7.84(\mathrm{~d}, J=7.5 \mathrm{~Hz}, 2 \mathrm{H}), 7.69(\mathrm{t}, J=7.5 \mathrm{~Hz}, 1 \mathrm{H}), 7.58(\mathrm{t}, J=7.5 \mathrm{~Hz}, 2 \mathrm{H}), 5.90$ 
(d, $J=1 \mathrm{~Hz}, 1 \mathrm{H}), 5.68(\mathrm{br} \mathrm{d}, J=1.5 \mathrm{~Hz}, 1 \mathrm{H}), 5.01(\mathrm{~s}, 1 \mathrm{H}), 4.04(\mathrm{~d}, J=14 \mathrm{~Hz}, 1 \mathrm{H}), 3.92$

(d, $J=14 \mathrm{~Hz}, 1 \mathrm{H}), 3.87$ (d, $J=11.5 \mathrm{~Hz}, 1 \mathrm{H}), 3.73(\mathrm{~d}, J=11.5 \mathrm{~Hz}, 1 \mathrm{H}), 3.72-3.66$ (br d, $1 \mathrm{H}), 2.57-2.53(\mathrm{~m}, 1 \mathrm{H}), 2.19$ (ddd, $J=1.5,8,15 \mathrm{~Hz}, 1 \mathrm{H}), 1.86(\mathrm{~d}, J=1 \mathrm{~Hz}, 3 \mathrm{H}), 1.71$ (dt, $J=6,6,15 \mathrm{~Hz}, 1 \mathrm{H}), 1.58(\mathrm{~s}, 3 \mathrm{H}), 1.56(\mathrm{~s}, 3 \mathrm{H}), 1.07$ (s, 3H), 1.00 (s, 3H), 0.97 (d, $J=$ $7 \mathrm{~Hz}, 3 \mathrm{H}), 0.81(\mathrm{dd}, J=8.5,11.5 \mathrm{~Hz}, 1 \mathrm{H}), 0.71-0.65(\mathrm{~m}, 1 \mathrm{H}) ;{ }^{13} \mathrm{C} \mathrm{NMR}(100 \mathrm{MHz}$, $\left.\mathrm{CDCl}_{3}\right) \delta$ 206.7, 138.0, 136.7, 135.0, 134.1, 131.5, 129.5, 129.4, 129.0, 114.9, 96.5, 89.0, $74.3,72.6,61.4,45.7,39.5,30.9,28.6,27.0,26.7,23.7,23.3,22.8,18.1,16.3,15.8$ LRMS (EI) $m / z 535.3\left(\mathrm{~m}+\mathrm{Na}^{+}\right)$.

\section{Preparation of diene 23'.}

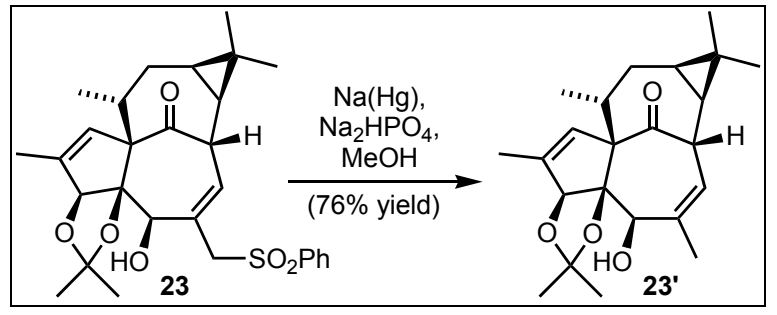

Diene 23'. Anhydrous methanol $(1.5 \mathrm{~mL})$ was added to a flask containing allylic sulfone $\mathbf{2 3}$ ( $9 \mathrm{mg}, 17 \mu \mathrm{mol}$ ) and allowed to stir for $30 \mathrm{~min}$, until dissolution was complete. $\mathrm{Na}_{2} \mathrm{HPO}_{4}\left(40 \mathrm{mg}, 280 \mu \mathrm{mol}, 15\right.$ equiv) was added and the solution was cooled to $-20^{\circ} \mathrm{C}$ in a methanol $/ \mathrm{NaCl} /$ ice bath. To this suspension was added $6 \% \mathrm{Na}(\mathrm{Hg})(160 \mathrm{mg}, \sim 400$ $\mu \mathrm{mol} \mathrm{Na}, \sim 25$ equiv) at once, and the suspension was allowed to warm to $-10^{\circ} \mathrm{C}$ over 25 minutes, at which point $\mathrm{H}_{2} \mathrm{O}$ and ether were added to quench the reaction. The biphasic solution was allowed to stir at room temperature until no more gas was evolved. The organic phase was separated, washed with water and brine, dried over $\mathrm{MgSO}_{4}$, and concentrated in vacuo. The residual oil was subjected to silica gel chromatography (19:1 hexanes:EtOAc eluent) to afford diene $\mathbf{2 3}^{\prime}$ ( $5 \mathrm{mg}, \mathbf{7 6} \%$ ) as a colorless oil. 
Diene 23': FTIR (thin film/NaCl) 3480 (br), 2923 (s), 1722 (s), 1456 (m), 1381 (s), $1024(\mathrm{~s}), 734(\mathrm{~m}) \mathrm{cm}^{-1} ;{ }^{1} \mathrm{H}$ NMR $\left(400 \mathrm{MHz}, \mathrm{CDCl}_{3}\right) \delta 5.84(\mathrm{br} \mathrm{s}, 1 \mathrm{H}), 5.82(\mathrm{~d}, J=$ $0.8 \mathrm{~Hz}, 1 \mathrm{H}), 4.68(\mathrm{~s}, 1 \mathrm{H}), 4.02(\mathrm{dd}, J=5,11 \mathrm{~Hz}, 1 \mathrm{H}), 3.79(\mathrm{~d}, J=8 \mathrm{~Hz}), 2.64(\mathrm{~m}, 1 \mathrm{H})$, $2.46(\mathrm{~d}, J=8 \mathrm{~Hz}, 1 \mathrm{H}), 2.15(\mathrm{ddd}, J=2.5,7,15 \mathrm{~Hz}, 1 \mathrm{H}), 1.84(\mathrm{br} \mathrm{s}, 3 \mathrm{H}), 1.81(\mathrm{~s}, 3 \mathrm{H})$, $1.70(\mathrm{dt}, J=6.5,6.5,15.5 \mathrm{~Hz}, 1 \mathrm{H}), 1.54(\mathrm{~s}, 3 \mathrm{H}), 1.14(\mathrm{~s}, 3 \mathrm{H}), 1.03(\mathrm{~s}, 3 \mathrm{H}), 0.98(\mathrm{~d}, J=$ $6.8 \mathrm{~Hz}, 3 \mathrm{H}), 1.0-0.9(\mathrm{~m}, 1 \mathrm{H}), 0.67(\mathrm{dd}, J=7,15 \mathrm{~Hz}, 1 \mathrm{H}) ;{ }^{13} \mathrm{C} \mathrm{NMR}\left(100 \mathrm{MHz}, \mathrm{CDCl}_{3}\right) \delta$ $207.6,137.7,137.0,130.9,126.2,114.0,96.5,89.8,75.9,75.0,44.3,38.8,31.8,28.9$, 28.1, 27.2, 24.0, 23.6, 23.5, 23.3, 18.2, 15.6, 15.5; LRMS (EI) $m / z 395.3\left(\mathrm{~m}+\mathrm{Na}^{+}\right)$.

\section{Preparation of 20-deoxyingenol (24).}

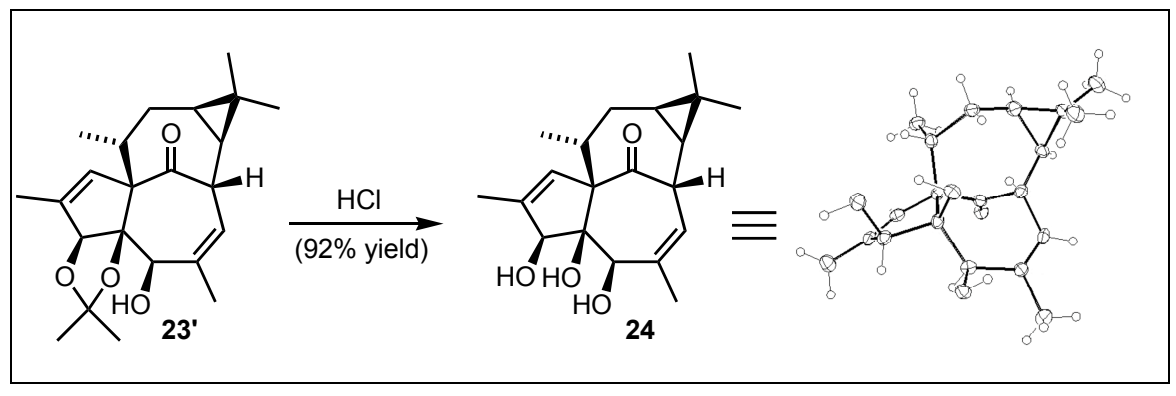

20-Deoxyingenol (24). To an open flask containing acetonide $\mathbf{2 3}^{\prime}$ (5 mg, $13 \mu \mathrm{mol})$ was added a solution of $1: 1 \mathrm{THF} / 1 \mathrm{~N} \mathrm{HCl}(1 \mathrm{~mL}$ total), and allowed to stir at room temperature. A white precipitate gradually appeared over $5 \mathrm{hrs,} \mathrm{at} \mathrm{which} \mathrm{point} \mathrm{the}$ reaction mixture was poured into aq. $\mathrm{NaHCO}_{3}$ and extracted with EtOAc. The organic phase was washed with brine, dried over $\mathrm{MgSO}_{4}$, and concentrated in vacuo, to give a white solid. The solid was rinsed twice with $\mathrm{CH}_{2} \mathrm{Cl}_{2}(1 \mathrm{~mL}$ total $)$ to give pure 20deoxyingenol (24) as a white precipitate. The $\mathrm{CH}_{2} \mathrm{Cl}_{2}$ rinse was chromatographed on silica gel (6:1 $\mathrm{CH}_{2} \mathrm{Cl}_{2}:$ EtOAc eluent), and the purified fractions were recombined with the precipitate. Further crystallization from 1:1 $\mathrm{CH}_{2} \mathrm{Cl}_{2}$ :EtOAc afforded 20-deoxyingenol 
$\mathbf{( 2 5}, 4 \mathrm{mg}, \mathbf{9 2} \%)$ as colorless crystals, upon which X-Ray diffraction analysis was performed (see cif file).

20-Deoxyingenol (25): m.p.: 208-209 ${ }^{\circ} \mathrm{C}$ (decomp); FTIR (thin film/NaCl) 3400 (br), 2924 (s), 1708 (m), 1461 (m), 1377 (m), 1017 (w) cm ${ }^{-1}$; ${ }^{1} \mathrm{H}$ NMR (500 MHz, $\left.\mathrm{C}_{5} \mathrm{D}_{5} \mathrm{~N}\right) \delta 6.28(\mathrm{~s}, 1 \mathrm{H}), 5.93(\mathrm{br} \mathrm{d}, J=5 \mathrm{~Hz}, 1 \mathrm{H}), 5.07(\mathrm{~s}, 1 \mathrm{H}), 5.05-4.95(\mathrm{br} \mathrm{s}, 3 \mathrm{H}), 4.65$ (br d, $J=10 \mathrm{~Hz}, 1 \mathrm{H}), 3.91(\mathrm{~s}, 1 \mathrm{H}), 2.85-2.82(\mathrm{~m}, 1 \mathrm{H}), 2.69-2.64(\mathrm{~m}, 1 \mathrm{H}), 2.00(\mathrm{~s}, 3 \mathrm{H})$, $1.91(\mathrm{~s}, 3 \mathrm{H}), 1.76(\mathrm{ddd}, J=5,6,15.5 \mathrm{~Hz}, 1 \mathrm{H}), 1.26(\mathrm{~s}, 3 \mathrm{H}), 1.2(\mathrm{~m}, 1 \mathrm{H}), 1.17$ (d, $J=7.5$ $\mathrm{Hz}, 3 \mathrm{H}), 1.07$ (s, 3H), 0.80-0.74 (m, 1H); ${ }^{13} \mathrm{C}$ NMR $\left(125 \mathrm{MHz}, \mathrm{C}_{5} \mathrm{D}_{5} \mathrm{~N}\right) \delta$ 207.9, 140.8, $140.7,129.8,86.3,80.9,77.3,73.6,44.8,40.4,31.7,29.0,24.6,24.5,23.9,23.3,17.9$, 16.2, 16.1; LRMS (EI) $m / z 355.2\left(\mathrm{~m}+\mathrm{Na}^{+}\right)$.

\section{Preparation of ingenol (1).}

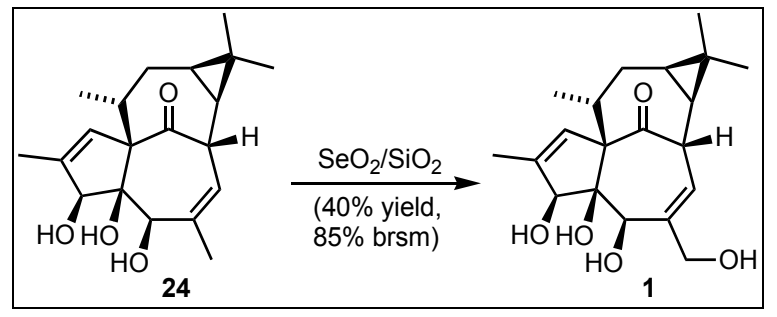

Ingenol (1). To a 4 dram screw cap vial (VWR Scientific) containing 20deoxyingenol (24, $4 \mathrm{mg}, 12 \mu \mathrm{mol})$ was added THF $(1.5 \mathrm{~mL})$ and 5\% silica supported selenium dioxide (112 mg, $50 \mu \mathrm{mol}, 4$ equiv). The vial was sealed and heated in a sand bath to $80^{\circ} \mathrm{C}$ for $2 \mathrm{hrs}$, at which point the reaction was cooled, filtered through celite, and concentated in vacuo. Flash chromatography $\left(3: 1 \rightarrow 1: 1 \mathrm{CH}_{2} \mathrm{Cl}_{2}\right.$ :EtOAc eluent) afforded starting material 24 (2.2 $\mathrm{mg}, 65 \%)$, and ingenol (1, 1.6 mg, 40\%) as a colorless oil.

Ingenol (1): FTIR (thin film/NaCl) 3380 (br), 2922 (s), 2850 (s), 1711 (m), 1462 (m) $\mathrm{cm}^{-1} ;{ }^{1} \mathrm{H}$ NMR $\left(500 \mathrm{MHz}, \mathrm{CDCl}_{3}\right) \delta 6.06(\mathrm{br} \mathrm{d}, J=5.5 \mathrm{~Hz}, 1 \mathrm{H}), 5.95(\mathrm{~d}, J=1.5 \mathrm{~Hz}$, 
1H), $4.41(\mathrm{~s}, 1 \mathrm{H}), 4.18$ (d, $J=13 \mathrm{~Hz}, 1 \mathrm{H}), 4.12$ (d, $J=13 \mathrm{~Hz}, 1 \mathrm{H}), 4.13-4.09$ (m, 1H), $4.10(\mathrm{~s}, 1 \mathrm{H}), 3.81(\mathrm{~s}, 1 \mathrm{H}), 2.35-2.25(\mathrm{~m}, 2 \mathrm{H}), 1.86(\mathrm{~d}, J=1.5 \mathrm{~Hz}, 3 \mathrm{H}), 1.76(\mathrm{dt}, J=5,5$, $15.5 \mathrm{~Hz}, 1 \mathrm{H}), 1.12$ (s, 3H), 1.06 (s, 3H), 0.97 (d, $J=6.5 \mathrm{~Hz}, 3 \mathrm{H}), 0.9$ (m, 1H), 0.72-0.68 (m, 1H); ${ }^{13} \mathrm{C}$ NMR $\left(125 \mathrm{MHz}, \mathrm{CDCl}_{3}\right) \delta 207.2,140.5,139.0,130.4,127.6,84.6,80.8$, 75.6, 72.7, 67.3, 44.3, 40.1, 31.1, 28.7, 24.2, 23.3, 23.2, 17.5, 15.7, 15.7; LRMS (EI) $\mathrm{m} / \mathrm{z}$ $371.3\left(\mathrm{~m}+\mathrm{Na}^{+}\right)$. 